\title{
Induced quasicocycles on groups with hyperbolically embedded subgroups
}

\author{
Michael Hull \\ DENIS OSIN
}

\begin{abstract}
Let $G$ be a group, $H$ a hyperbolically embedded subgroup of $G, V$ a normed $G$-module, $U$ an $H$-invariant submodule of $V$. We propose a general construction which allows to extend 1-quasicocycles on $H$ with values in $U$ to 1-quasicocycles on $G$ with values in $V$. As an application, we show that every group $G$ with a nondegenerate hyperbolically embedded subgroup has $\operatorname{dim} H_{b}^{2}\left(G, \ell^{p}(G)\right)=\infty$ for $p \geq 1$. This covers many previously known results in a uniform way. Applying our extension to quasimorphisms and using Bavard duality, we also show that hyperbolically embedded subgroups are undistorted with respect to the stable commutator length.
\end{abstract}

20F65, 20F67, 20J06, 43A15, 57M07

\section{Introduction}

Let $\mathbb{F}$ be a subfield of $\mathbb{C}$. All modules in this paper are left, all vector spaces are over $\mathbb{F}$. For a discrete group $G$, by a normed $G$-module we mean a normed vector space $V$ endowed with a (left) action of the group $G$ by isometries. Given a subgroup $H \leq G$, by an $H$-submodule of a $G$-module $V$ we mean any $H$-invariant subspace of $V$ with the induced action of $H$.

Let $V$ be a normed $G$-module. Recall that a map $q: G \rightarrow V$ is called a 1-quasicocycle if there exists a constant $\varepsilon>0$ such that for every $f, g \in G$ we have

$$
\|q(f g)-q(f)-f q(g)\| \leq \varepsilon .
$$

The $\mathbb{F}$-vector space of all 1-quasicocycles on $G$ with values in $V$ is denoted by $Q Z^{1}(G, V)$.

The study of 1-quasicocycles is partially motivated by the fact that the kernel of the comparison map $H_{b}^{2}(G, V) \rightarrow H^{2}(G, V)$ from the second bounded cohomology to the ordinary second cohomology with coefficients in $V$ can be identified with the quotient $Q Z^{1}(G, V) /\left(\ell^{\infty}(G, V)+Z^{1}(G, V)\right)$, where $\ell^{\infty}(G, V)$ and $Z^{1}(G, V)$ are 
the subspaces of uniformly bounded maps and cocycles, respectively. In the last decade, techniques based on 1-quasicocycles and bounded cohomology have led to new breakthroughs in the study of rigidity of group von Neumann algebras, measure equivalence and orbit equivalence of groups, and low dimensional topology (see Calegari [7], Chifan and Sinclair [9], Monod [19], Popa [24] and references therein).

The main goal of this paper is to address the following "extension problem": Under what conditions can a 1-quasicocycle on a subgroup $H \leq G$ be extended to the whole group $G$ ?

Below we describe few known results in this direction.

Example 1.1 (Counting quasimorphisms) If $V=\mathbb{R}$ with the trivial action of $G$, 1-quasicocycles on $G$ with values in $V$ are called quasimorphisms. The classical examples are counting quasimorphisms of free groups introduced by Brooks [6]. Let $F$ be a free group with a basis $S$ and let $w$ be a reduced word in $S \cup S^{-1}$. Given an element $f \in F$, denote by $c_{w}(g)$ the number of disjoint copies of $w$ in the reduced representative of $g$. Then $h_{w}=c_{w}-c_{w^{-1}}$ defines a quasimorphism $F \rightarrow \mathbb{R}$ (Brooks [6]). Observe that $h_{w}(g)$ extends the obvious cocycle (ie, homomorphism) $H \rightarrow \mathbb{R}$ of the cyclic subgroup $H=\langle w\rangle \leq F$ that sends $w^{n}$ to $n$ for all $n \in \mathbb{Z}$.

This construction was further developed by Epstein and Fujiwara [11] and later by Bestvina and Fujiwara [4], who generalized it to the cases of hyperbolic groups and groups acting weakly properly discontinuous on hyperbolic spaces, respectively.

Recall that a 1-quasicocycle $q \in Q Z^{1}(G, V)$ is called antisymmetric if

$$
q\left(g^{-1}\right)=-g^{-1} q(g)
$$

for every $g \in G$. The next example is essentially due to Thom (cf [26, Lemma 5.1]).

Example 1.2 (Extending antisymmetric 1-quasicocycles to free products) Let $G=$ $H_{1} * H_{2}$, let $V$ be a normed $G$-module, and let $U_{i}$ be an $H_{i}$-submodule of $G$, $i=1,2$. Then any antisymmetric 1 -quasicocycles $q_{i} \in Q Z^{1}\left(H_{i}, U_{i}\right), i=1,2$, can be naturally extended to a 1-quasicocycle $G \rightarrow V$ using the normal form of elements of free products. That is, suppose that $g \in G$ has the normal form

$$
g=h_{1} k_{1} \cdots h_{n} k_{n},
$$

where $h_{i} \in H_{1}, k_{i} \in H_{2}$ for $i=1, \ldots, n$, and $k_{1}, h_{2}, \ldots, k_{n-1}, h_{n}$ are nontrivial. Let

$$
q(g)=q_{1}\left(h_{1}\right)+h_{1} q_{2}\left(k_{1}\right)+h_{1} k_{1} q_{1}\left(h_{2}\right)+\cdots+h_{1} k_{1} \cdots h_{n} q_{2}\left(k_{n}\right) .
$$

Checking that $q \in Q Z^{1}(G, V)$ is easy. It is essential here that $q_{1}$ and $q_{2}$ are antisymmetric (see Remark 4.8). 
Example 1.3 (No general extension construction exists) It is well-known and easy to prove that every quasimorphism on an amenable group decomposes as a sum of a homomorphism and a bounded map (Calegari [7]). This easily implies that if $G$ is amenable and $H=\langle h\rangle \leq[G, G]$ is an infinite cyclic subgroup, then the natural homomorphism $H \rightarrow \mathbb{R}$ defined by $h^{n} \mapsto n$ does not extend to any quasimorphism of $G$.

In this paper we prove an extension theorem which can be thought of as a generalization of Examples 1.1 and 1.2. In fact, our construction is similar to Example 1.2, but the proof is much more involved. We state here a simplified version of our main result and refer to Theorem 4.2 for the full generality. For a group $G$ and a normed $G$-module $V$, let $Q Z_{a s}^{1}(G, V)$ denote the subspace of all antisymmetric 1-quasicocycles on $G$ with coefficients in $V$.

Theorem 1.4 Let $G$ be a group, $H$ a hyperbolically embedded subgroup of $G, V$ a normed $G$-module, $U$ an $H$-submodule of $V$. Then there exists a linear map

$$
\iota: Q Z_{a s}^{1}(H, U) \rightarrow Q Z_{a s}^{1}(G, V)
$$

such that for any $q \in Q Z_{\text {as }}^{1}(H, U)$, we have $\left.\iota(q)\right|_{H} \equiv q$.

It is well-known and easy to prove that every 1-quasicocycle is antisymmetric up to a bounded perturbation (see Lemma 2.5). In the notation of Theorem 1.4, this gives the following.

Corollary 1.5 There exists a linear map $\varkappa: Q Z^{1}(H, U) \rightarrow Q Z^{1}(G, V)$ such that for any $q \in Q Z^{1}(H, U),\left.\varkappa(q)\right|_{H} \in Q Z^{1}(H, U)$ and

$$
\sup _{h \in H}\|\varkappa(q)(h)-q(h)\|<\infty .
$$

The notion of a hyperbolically embedded subgroup of a group was introduced in Dahmani, Guirardel and Osin [10] and encompasses many examples of algebraic and geometric nature. We discuss some of them here and refer to the next section and the same work for the definition and details.

(a) Let $G$ be any group and let $H \leq G$ be a finite subgroup or $H=G$. Then $H$ is hyperbolically embedded in $G$. In what follows these cases are referred to as degenerate.

(b) Let $G$ be a group hyperbolic relative to a collection of peripheral subgroups $\left\{H_{\lambda}\right\}_{\lambda \in \Lambda}$. Then every peripheral subgroup is hyperbolically embedded in $G$. In particular, if $G=H_{1} * H_{2}$, then $H_{1}$ and $H_{2}$ are hyperbolically embedded in $G$. 
(c) Let $G$ be a relatively hyperbolic group and let $g$ be a loxodromic element. Then $g$ is contained in the unique maximal virtually cyclic subgroup $E(g)$ of $G$ and $E(g)$ is hyperbolically embedded in $G$ (Osin [22]). In particular, this holds for every infinite order element $g$ of a hyperbolic group $G$.

(d) More generally, let $G$ be a group acting on a hyperbolic space and containing a loxodromic element $g$ that satisfies the Bestvina-Fujiwara WPD condition (see Bestvina and Fujiwara [4] or Dahmani et al [10] for the definition). Then $g$ is contained in the unique maximal virtually cyclic subgroup $E(g)$ of $G$ and $E(g)$ is hyperbolically embedded in $G$ (Dahmani et al [10, Theorem 6.8]). This general result applies in the following cases: $\left(\mathrm{d}_{1}\right) G$ is the mapping class group of a punctured closed orientable surface and $g$ is a pseudo-Anosov element (Bestvina and Fujiwara [4]); $\left(\mathrm{d}_{2}\right) G=$ $\operatorname{Out}\left(F_{n}\right)$ and $g$ is a fully irreducible automorphism (Bestvina and Feighn [3]).

(e) Similarly to the previous example, let $G$ be a group acting properly on a proper CAT( 0$)$ space and let $g$ be a rank 1 element. Then $g$ is contained in the unique maximal virtually cyclic subgroup $E(g)$ of $G$ and $E(g)$ is hyperbolically embedded in $G$ (Sisto [25]).

Example 1.6 (cf Bestvina and Fujiwara [4], Calegari and Fujiwara [8]) Let us illustrate our theorem by extending quasimorphisms in the case when $G$ and $g$ are as in examples (c), (d) or (e) above. It is well known and easy to prove that every infinite virtually cyclic group is either finite-by-(infinite cyclic) or finite-by-(infinite dihedral). If $E(g)$ is of the former type, there exists a homomorphism $q: E(g) \rightarrow \mathbb{R}$ that extends the natural map $g^{n} \rightarrow n$. By our theorem, $q$ extends to a quasimorphism of $G$, which can be thought of as a generalization of the Brooks' counting quasimorphism. In particular, such quasimorphisms can always be constructed if $G$ has no involutions.

On the other hand, if $E(g)$ is finite-by-(infinite dihedral), then it is easy to show that there exists $a \in G$ and $n \in \mathbb{N}$ such that

$$
a^{-1} g^{n} a=g^{-n} \text {. }
$$

This equality implies that every quasimorphism $E(g) \rightarrow \mathbb{R}$ is bounded. Thus no analogue of the counting quasimorphism exists in this case.

In Section 3, we develop the main idea in the construction of our extension, which is the notion of separating cosets of a subgroup $H$ which is hyperbolically embedded in $G$. This allows use to associate a canonical, finite set of $H$-cosets to each $g \in G$, and to each such coset a finite collection of $h \in H$. This is essentially what is given by the normal forms of elements in Example 1.2, and we are then able to extend quasicocycles 
in a similar manner. The main technical tool in proving that our extension actually gives a quasicocycle is the decomposition of the separating cosets of a triangle in Lemma 3.9.

In Section 5, we obtain some other corollaries of our main result. Recall that the class $\mathcal{C}_{\text {reg }}$ of Monod-Shalom is the class of groups for which $H_{b}^{2}\left(G, \ell^{2}(G)\right) \neq 0$. This definition was proposed as cohomological characterization of the notion of "negative curvature" in group theory (Monod and Shalom [20]). Monod and Shalom [21] develop a rich rigidity theory with respect to measure equivalence and orbit equivalence of actions of groups in $\mathcal{C}_{\text {reg }}$. These results have a variety of applications to measurable group theory, ergodic theory and von Neumann algebras.

Another similar class of groups is the class $\mathcal{D}_{\text {reg }}$ introduced by Thom [26]. $G \in \mathcal{D}_{\text {reg }}$ if $G$ is nonamenable and there exists some $q \in Q Z^{1}\left(G, \ell^{2}(G)\right)$ which is unbounded. Thom proved rigidity results about the subgroup structure of groups in $\mathcal{D}_{\text {reg }}$ and showed that this class is closely related to $\mathcal{C}_{\text {reg }}$. However neither inclusion is known to hold between these two classes.

Let $\mathcal{X}$ denote the class of groups with nondegenerate hyperbolically embedded subgroups. Using Corollary 1.5 and the fact that every group $G \in \mathcal{X}$ contains a virtually free (but not virtually cyclic) hyperbolically embedded subgroup (Dahmani et al [10]), we prove the following.

Corollary 1.7 For any $G \in \mathcal{X}$, the dimension of the kernel of the comparison map $H_{b}^{2}\left(G, \ell^{p}(G)\right) \rightarrow H^{2}\left(G, \ell^{p}(G)\right)$ is infinite. In particular, $\mathcal{X} \subseteq \mathcal{C}_{\text {reg }} \cap \mathcal{D}_{\text {reg }}$.

This corollary recovers several previously known results in a uniform way. For example, this was known for hyperbolic groups (Mineyev, Monod and Shalom [17]) and more generally groups acting nonelementary and acylindrically on hyperbolic spaces (Hamenstädt [14]), groups acting properly on proper CAT(0) spaces and containing a rank 1 isometry (Hamenstädt [15]), and $\operatorname{Out}\left(F_{n}\right)$ for $n \geq 2$ (Hamenstädt [13]). All of these groups belong to $\mathcal{X}$ (Dahmani et al [10]).

At the final stage of our work we learned that Bestvina, Bromberg and Fujiwara [2] independently and simultaneously proved that the dimension of the kernel of the comparison map $H_{b}^{2}(G, E) \rightarrow H^{2}(G, E)$ is infinite for any group acting nonelementary on a hyperbolic space and containing a WPD loxodromic isometry and any uniformly convex Banach $G$-module $E$. In fact, the class of groups acting nonelementary on a hyperbolic space and containing a WPD loxodromic isometry coincides with our class $\mathcal{X}$ (see Dahmani et al [10, Theorem 6.8 and Corollary 6.10]). Thus the result of Bestvina, Bromberg and Fujiwara [2] is stronger than Corollary 1.7.

As another application, we show that hyperbolically embedded subgroups are undistorted with respect to the stable commutator length, scl. For the definition of scl we 
refer to Section 5. Given a group $G$ and a subgroup $H \leq G$ it is straightforward to see that $\operatorname{scl}_{G}(h) \leq \operatorname{scl}_{H}(h)$ for any $h \in[H, H]$, where $\operatorname{scl}_{G}$ and $\operatorname{scl}_{H}$ are the stable commutator lengths on $[G, G]$ and $[H, H]$, respectively.

On the other hand, recall that every torsion free group $H$ can be embedded in a group $G$ where every element is a commutator (see Lyndon and Schupp [16, Theorem 8.1] or Osin [23] for a finitely generated version of such an embedding). In particular, $\operatorname{scl}_{G}$ vanishes on $G$, while $\operatorname{scl}_{H}$ can be unbounded on $[H, H]$. Thus, in general, there is no upper bound on $\mathrm{scl}_{H}$ in terms of $\operatorname{scl}_{G}$. In what follows, we say that $H$ is undistorted in $G$ with respect to the stable commutator length if there exists a constant $B$ such that for every $h \in[H, H]$, we have $\operatorname{scl}_{H}(h) \leq B \operatorname{scl}_{G}(h)$.

Using Theorem 1.4 and the Bavard duality, we obtain the following.

Corollary 1.8 Let $G$ be a group, $H$ a hyperbolically embedded subgroup of $G$. Then $H$ is undistorted in $G$ with respect to the stable commutator length.

Even the following particular cases seem new. Recall that a subgroup $H \leq G$ is almost malnormal if $\left|H^{g} \cap H\right|<\infty$ for every $g \in G \backslash H$.

Corollary 1.9 Every almost malnormal quasiconvex subgroup of a hyperbolic group is undistorted with respect to the stable commutator length. In particular, so is every finitely generated malnormal subgroup of a free group.

In Section 5 we show that the almost malnormality condition can not be omitted even for free groups (see Remark 5.8).

\section{Preliminaries}

Notation and conventions In this paper we allow length functions and metrics to take infinite values. In particular, the word length $|\cdot|_{S}$ on a group $G$ corresponding to a (not necessary generating) set $S$ is defined by letting $|g|_{S}$ be the length of a shortest word in $S \cup S^{-1}$ representing $g$ if $g \in\langle S\rangle$ and $|g|_{S}=\infty$ otherwise. The corresponding metric on $G$ is denoted by $\mathrm{d}_{S}$; thus $\mathrm{d}_{S}(f, g)=\left|f^{-1} g\right|_{S}$.

By a path $p$ in a (Cayley) graph we always mean a combinatorial path; we denote the label of $p$ by $\mathbf{L a b}(p)$ and we denote the origin and terminus of $p$ by $p_{-}$and $p_{+}$ respectively.

For the rest of the paper, we will refer to 1-quasicocycles simply as quasicocycles. 
Hyperbolically embedded subgroups Let $G$ be a group, $\left\{H_{\lambda}\right\}_{\lambda \in \Lambda}$ a collection of subgroups of $G$. Let

$$
\mathcal{H}=\bigsqcup_{\lambda \in \Lambda} H_{\lambda}
$$

Given a subset $X \subseteq G$ such that $G$ is generated by $X$ together with the union of all $H_{\lambda}$ 's, we denote by $\Gamma(G, X \sqcup \mathcal{H})$ the Cayley graph of $G$ whose edges are labeled by letters from the alphabet $X \sqcup \mathcal{H}$. That is, two vertices $g, h \in G$ are connected by an edge going from $g$ to $h$ and labeled by $a \in X \sqcup \mathcal{H}$ if and only if $a$ represents the element $g^{-1} h$ in $G$. Note that some letters from $X \sqcup \mathcal{H}$ may represent the same element in $G$, in which case $\Gamma(G, X \sqcup \mathcal{H})$ has multiple edges corresponding to these letters.

We think of the Cayley graphs $\Gamma\left(H_{\lambda}, H_{\lambda}\right)$ as (complete) subgraphs of $\Gamma(G, X \sqcup \mathcal{H})$. For every $\lambda \in \Lambda$, we introduce a relative metric $\widehat{\mathrm{d}}_{\lambda}: H_{\lambda} \times H_{\lambda} \rightarrow[0,+\infty]$ as follows. Given $h, k \in H_{\lambda}$, let $\widehat{\mathrm{d}}_{\lambda}(h, k)$ be the length of a shortest path in $\Gamma(G, X \sqcup \mathcal{H})$ that connects $h$ to $k$ and has no edges in $\Gamma\left(H_{\lambda}, H_{\lambda}\right)$. If no such a path exists, we set $\widehat{\mathrm{d}}_{\lambda}(h, k)=\infty$. Clearly $\widehat{\mathrm{d}}_{\lambda}$ satisfies the triangle inequality. In case the collection consists of a single subgroup $H \leq G$, we denote the corresponding relative metric on $H$ simply by $\widehat{d}$.

Definition 2.1 Let $G$ be a group, $X$ a (not necessary finite) subset of $G$. We say that a collection of subgroups $\left\{H_{\lambda}\right\}_{\lambda \in \Lambda}$ of $G$ is hyperbolically embedded in $G$ with respect to $X$ (we write $\left\{H_{\lambda}\right\}_{\lambda \in \Lambda} \hookrightarrow_{h}(G, X)$ ) if the following conditions hold.

(a) The group $G$ is generated by $X$ together with the union of all $H_{\lambda}$ and the Cayley graph $\Gamma(G, X \sqcup \mathcal{H})$ is hyperbolic.

(b) For every $\lambda \in \Lambda,\left(H_{\lambda}, \widehat{\mathrm{d}}_{\lambda}\right)$ is a locally finite metric space; that is, any ball of finite radius in $H_{\lambda}$ contains finitely many elements.

Further we say that $\left\{H_{\lambda}\right\}_{\lambda \in \Lambda}$ is hyperbolically embedded in $G$ and write

$$
\left\{H_{\lambda}\right\}_{\lambda \in \Lambda} \hookrightarrow_{h} G
$$

if $\left\{H_{\lambda}\right\}_{\lambda \in \Lambda} \hookrightarrow_{h}(G, X)$ for some $X \subseteq G$.

Example 2.2 (a) Let $G$ be any group. Then $G \hookrightarrow_{h} G$. Indeed take $X=\varnothing$. Then the Cayley graph $\Gamma(G, X \sqcup H)$ has diameter 1 and $d\left(h_{1}, h_{2}\right)=\infty$ whenever $h_{1} \neq h_{2}$. Further, if $H$ is a finite subgroup of a group $G$, then $H \hookrightarrow_{h} G$. Indeed $H \hookrightarrow_{h}(G, X)$ for $X=G$. These cases are referred to as degenerate. In what follows we are only interested in nondegenerate examples. 
(b) Let $G=H \times \mathbb{Z}, X=\{x\}$, where $x$ is a generator of $\mathbb{Z}$. Then $\Gamma(G, X \sqcup H)$ is quasiisometric to a line and hence it is hyperbolic. However $\widehat{\mathrm{d}}\left(h_{1}, h_{2}\right) \leq 3$ for every $h_{1}, h_{2} \in H$. Indeed let $\Gamma_{H}$ denote the Cayley graph $\Gamma(H, H \backslash\{1\})$. In the shift $x \Gamma_{H}$ of $\Gamma_{H}$ there is an edge (labeled by $h_{1}^{-1} h_{2} \in H$ ) connecting $h_{1} x$ to $h_{2} x$, so there is a path of length 3 connecting $h_{1}$ to $h_{2}$ and having no edges in $\Gamma_{H}$ (see Figure 1). Thus if $H$ is infinite, then $H \hookrightarrow_{h}(G, X)$. Moreover, a similar argument shows that $H \leftrightarrow{ }_{h} G$.

(c) Let $G=H * \mathbb{Z}, X=\{x\}$, where $x$ is a generator of $\mathbb{Z}$. In this case $\Gamma(G, X \sqcup H)$ is quasiisometric to a tree (see Figure 1) and $\widehat{\mathrm{d}}\left(h_{1}, h_{2}\right)=\infty$ unless $h_{1}=h_{2}$. Thus $H \hookrightarrow_{h}(G, X)$.
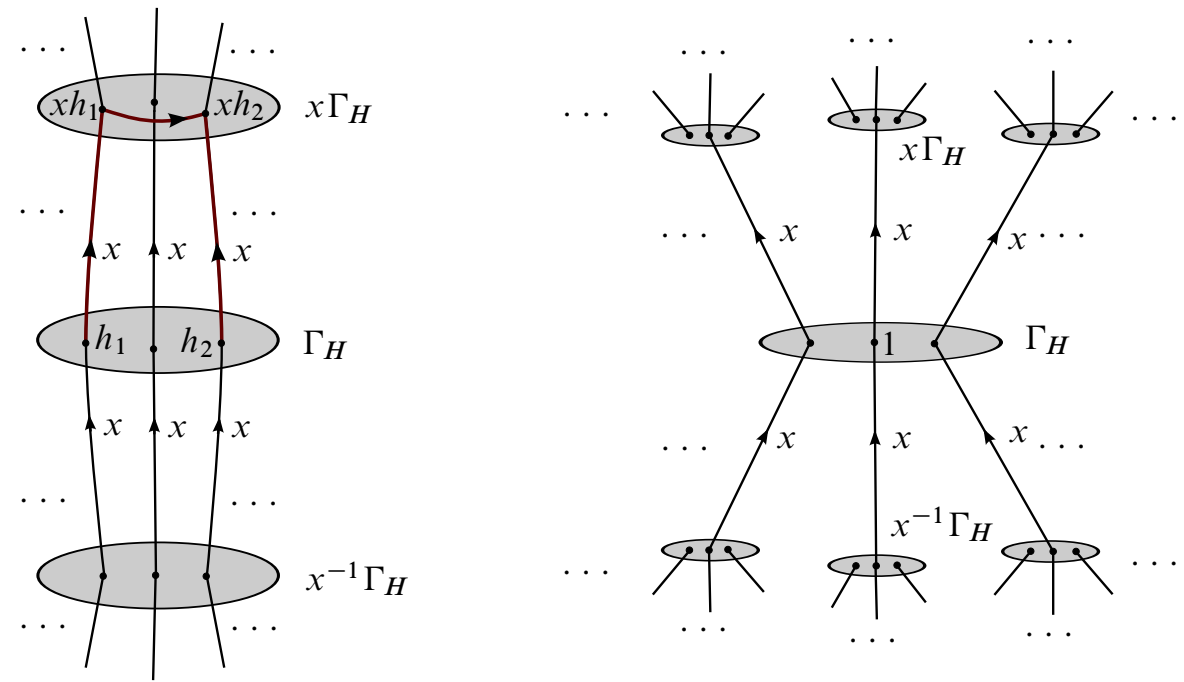

Figure 1: Cayley graphs $\Gamma(G, X \sqcup H)$ for $G=H \times \mathbb{Z}$ and $G=H * \mathbb{Z}$

It turns out that the relative metric $\widehat{\mathrm{d}}_{\lambda}$ can be realized as a word metric with respect to some finite set.

Lemma 2.3 (Dahmani et al [10, Lemma 4.10]) Let $\left\{H_{\lambda}\right\}_{\lambda \in \Lambda} \hookrightarrow_{h} G$. Then for each $\lambda \in \Lambda$, there exists a finite subset $Y_{\lambda} \subseteq H_{\lambda}$ such that $\widehat{\mathrm{d}}_{\lambda}$ is bi-Lipschitz equivalent to the word metric with respect to $Y_{\lambda}$. That is, for $h_{1}, h_{2} \in H_{\lambda}, \widehat{\mathrm{d}}_{\lambda}\left(h_{1}, h_{2}\right)$ is finite if and only if $\mathrm{d}_{Y_{\lambda}}\left(h_{1}, h_{2}\right)$ is, and the ratio $\widehat{\mathrm{d}}_{\lambda} / \mathrm{d}_{Y_{\lambda}}$ is uniformly bounded on $H_{\lambda} \times H_{\lambda}$ minus the diagonal. 


\section{Components Let}

$$
\left\{H_{\lambda}\right\}_{\lambda \in \Lambda} \hookrightarrow_{h}(G, X) .
$$

Let $q$ be a path in the Cayley graph $\Gamma(G, X \sqcup \mathcal{H})$. A (nontrivial) subpath $p$ of $q$ is called an $H_{\lambda}$-subpath, if the label of $p$ is a word in the alphabet $H_{\lambda} \backslash\{1\}$. An $H_{\lambda}$-subpath $p$ of $q$ is an $H_{\lambda}$-component if $p$ is not contained in a longer $H_{\lambda}$-subpath of $q$; if $q$ is a loop, we require in addition that $p$ is not contained in any longer $H_{\lambda}-$ subpath of a cyclic shift of $q$. Further by a component of $q$ we mean an $H_{\lambda}$-component of $q$ for some $\lambda \in \Lambda$.

Two $H_{\lambda}$-components $p_{1}, p_{2}$ of a path $q$ in $\Gamma(G, X \sqcup \mathcal{H})$ are called connected if there exists a path $c$ in $\Gamma(G, X \sqcup \mathcal{H})$ that connects some vertex of $p_{1}$ to some vertex of $p_{2}$, and $\mathbf{L a b}(c)$ is a word consisting only of letters from $H_{\lambda} \backslash\{1\}$. In algebraic terms this means that all vertices of $p_{1}$ and $p_{2}$ belong to the same left coset of $H_{\lambda}$. Note also that we can always assume that $c$ has length at most 1 as every nontrivial element of $H_{\lambda}$ is included in the set of generators.

It is convenient to extend the metric $\widehat{\mathrm{d}}_{\lambda}$ defined above to the whole group $G$ by assuming $\widehat{\mathrm{d}}_{\lambda}(f, g):=\widehat{\mathrm{d}}_{\lambda}\left(f^{-1} g, 1\right)$ if $f^{-1} g \in H_{\lambda}$ and $\widehat{\mathrm{d}}_{\lambda}(f, g)=\infty$ otherwise. One important technical tool is the following corollary of (a particular case of) Dahmani et al [10, Proposition 4.13].

Lemma 2.4 There exists a constant $C>0$ such that for any geodesic $n$-gon $p$ in $\Gamma(G, X \sqcup \mathcal{H})$ and any isolated component $a$ of $p$, we have $\widehat{\mathrm{d}}_{\lambda}\left(a_{-}, a_{+}\right) \leq C n$.

Proof Let $p=p_{1} \cdots p_{n}$, where $p_{1}, \ldots, p_{n}$ are geodesic. For definiteness, suppose that $a$ is a component of $p_{1}$, ie $p_{1}=q a r$. By Dahmani et al [10, Proposition 4.13] applied to the $(n+2)$-gon $\operatorname{qarp}_{2} \cdots p_{n}$, we have $\widehat{\mathrm{d}}_{\lambda}\left(a_{-}, a_{+}\right) \leq D(n+2) \leq 2 D n$, where $D$ is a constant independent of $n(D=D(1,0)$ in the notation of Dahmani et al [10, Proposition 4.13]). It remains to take any positive $C \geq 2 D$.

Quasicocycles For a quasicocycle $q \in Q Z^{1}(G, V)$ we define its defect $D(q)$ by

$$
D(q)=\sup _{f, g \in G}\|q(f g)-q(f)-f q(g)\| .
$$

Note that

$$
\|q(1)\|=\|q(1 \cdot 1)-q(1)-1 q(1)\| \leq D(q) .
$$

We will use the following elementary fact. 
Lemma 2.5 Let $G$ be a group, $V$ a $G$-module. Then there exists a linear map

$$
\alpha: Q Z^{1}(G, V) \rightarrow Q Z_{a s}^{1}(G, V)
$$

such that for every $q \in Q Z^{1}(G, V)$ we have

$$
\sup _{g \in G}\|\alpha(q)(g)-q(g)\|<D(q) .
$$

Proof Take $\alpha(q)(g)=\frac{1}{2}\left(q(g)-g q\left(g^{-1}\right)\right)$. Verifying all properties is straightforward. Indeed for every $g \in G$, we have

$$
\begin{aligned}
\|\alpha(q)(g)-q(g)\| & =\frac{1}{2}\left\|-q(g)-g q\left(g^{-1}\right)\right\| \\
& \leq \frac{1}{2}\left\|q(1)-q(g)-g q\left(g^{-1}\right)\right\|+\frac{1}{2}\|q(1)\| \\
& \leq D(q),
\end{aligned}
$$

where the last inequality uses (3). Further,

$$
\alpha(q)\left(g^{-1}\right)=\frac{1}{2}\left(q\left(g^{-1}\right)-g^{-1} q(g)\right)=\frac{1}{2} g^{-1}\left(g q\left(g^{-1}-q(g)\right)=-g^{-1} \alpha(q)(g) .\right.
$$

Bounded cohomology Recall the definition of the bounded cohomology of a (discrete) group $G$ with coefficients in an arbitrary normed $G$-module $V$. Let $C^{n}(G, V)$ be the vector space of $n$-cochains with coefficients in $V$, ie functions $G^{n} \rightarrow V$. The coboundary maps $d^{n}: C^{n}(G, V) \rightarrow C^{n+1}(G, V)$ are defined by the formula $d^{n} f\left(g_{1}, \ldots, g_{n+1}\right)=g_{1} f\left(g_{2}, \ldots, g_{n+1}\right)$

$$
\begin{aligned}
& +\sum_{i=1}^{n}(-1)^{i} f\left(g_{1}, \ldots, g_{i-1}, g_{i} g_{i+1}, g_{i+2}, \ldots, g_{n+1}\right) \\
& +(-1)^{n+1} f\left(g_{1}, \ldots, g_{n}\right) .
\end{aligned}
$$

Let $Z^{n}(G, V)$ and $B^{n}(G, V)$ denote the cocycles and coboundaries of this complex respectively; that is, $Z^{n}(G, V)=\operatorname{Ker} d^{n}$ and $B^{n}(G, V)=\operatorname{Im} d^{n-1}$ for $n>0$ and $B^{0}(G, V)=0$. Recall that the ordinary cohomology groups are defined by

$$
H^{n}(G, V):=Z^{n}(G, V) / B^{n}(G, V) .
$$

Restricting to the subspaces $C_{b}^{n}(G, V)$ of $C^{n}(G, V)$ consisting of functions whose image is bounded with respect to the norm on $V$, we get the complex of bounded cochains. Similarly let $Z_{b}^{n}(G, V)$ and $B_{b}^{n}(G, V)$ denote its cocycles and coboundaries. Then the group

$$
H_{b}^{n}(G, V):=Z_{b}^{n}(G, V) / B_{b}^{n}(G, V)
$$

is called the $n^{\text {th }}$-bounded cohomology group of $G$ with coefficients in $V$. 
Note that there is a natural map $c: H_{b}^{n}(G, V) \rightarrow H^{n}(G, V)$ which is induced by the inclusion map of the cochain complexes. This map is called the comparison map, and the kernel of $c$ is denoted $E H_{b}^{n}(G, V)$. The following lemma is proved in Monod [18] (see also Thom [26]) in the case when $V$ is a Banach space. The same proof works in the general case. We briefly sketch the argument for convenience of the reader.

Lemma 2.6 Let $G$ be a discrete countable group, $V$ a normed $G$-module. Then there exists an exact sequence

$$
0 \rightarrow \ell^{\infty}(G, V)+Z^{1}(G, V) \rightarrow Q Z^{1}(G, V) \stackrel{\delta}{\rightarrow} H_{b}^{2}(G, V) \stackrel{c}{\rightarrow} H^{2}(G, V),
$$

where $\ell^{\infty}(G, V)$ is the vector space of all uniformly bounded functions $G \rightarrow V$.

Proof We can identify $Q Z^{1}(G, V)$ with the subspace of 1-cochains $q$ for which $d^{1} q$ is uniformly bounded, that is $d^{1} q \in C_{b}^{2}(G, V)$. Since $d^{2} \circ d^{1} \equiv 0, d^{1} q$ is in fact a bounded 2-cocycle. Let $\delta: Q Z^{1}(G, V) \rightarrow H_{b}^{2}(G, V)$ denote the composition of $d^{1}$ and the natural quotient map $Z_{b}^{2}(G, V) \rightarrow H_{b}^{2}(G, V)$. Then $\delta q$ represents a trivial element of $H_{b}^{2}(G, V)$ if and only if $d^{1} q=d^{1} p$ for some bounded cochain $p$, which means $p \in \ell^{\infty}(G, V)$ and $q-p \in Z^{1}(G, V)$. Further if $q$ is a bounded 2-cocycle and $[q]_{b}:=q+B_{b}{ }^{2}(G, V) \in H_{b}^{2}(G, V)$ is in the kernel of $c$, then $q=d^{1} f$ for some 1-cochain $f$, which means $f \in Q Z^{1}(G, V)$ and $\delta f=[q]_{b}$.

\section{Separating cosets}

Throughout this section, we denote by $G$ a group with hyperbolically embedded collection of subgroups $\left\{H_{\lambda}\right\}_{\lambda \in \Lambda} \hookrightarrow_{h} G$. Let $X$ denote a subset of $G$ such that $\left\{H_{\lambda}\right\}_{\lambda \in \Lambda} \hookrightarrow_{h}(G, X)$. We also keep the notation $\mathcal{H}$ and $\Gamma(G, X \sqcup \mathcal{H})$ introduced in the previous section. By $\mathrm{d}_{X \cup \mathcal{H}}$ we denote the word metric on $G$ with respect to the subset $X \sqcup \mathcal{H}$. By a coset of a subgroup we always mean a left coset.

We begin by introducing the notion of a separating coset for a pair of elements $f, g \in G$, which plays a crucial role in our construction.

Definition 3.1 We say that a path $p$ in $\Gamma(G, X \sqcup \mathcal{H})$ penetrates a coset $x H_{\lambda}$ for some $\lambda \in \Lambda$ if $p$ decomposes as $p_{1} a p_{2}$, where $p_{1}, p_{2}$ are possibly trivial, $\left(p_{1}\right)_{+} \in x H_{\lambda}$, and $a$ is an $H_{\lambda}$-component of $p$. If, in addition, $\widehat{\mathrm{d}}_{\lambda}\left(a_{-}, a_{+}\right)>3 C$, where $C$ is the constant from Lemma 2.4, we say that $p$ essentially penetrates $x H_{\lambda}$. Note that if $p$ is geodesic, it penetrates every coset of $H_{\lambda}$ at most once; in this case the vertices $a_{-}$ and $a_{+}$are called the entrance and the exit points of $p$ in $x H_{\lambda}$ and are denoted by $p_{\text {in }}\left(x H_{\lambda}\right)$ and $p_{\text {out }}\left(x H_{\lambda}\right)$, respectively. 
Given two elements $f, g \in G$, we denote by $\mathcal{G}(f, g)$ the set of all geodesics in $\Gamma(G, X \sqcup \mathcal{H})$ going from $f$ to $g$. Further we say that a coset $x H_{\lambda}$ is $(f, g)$-separating if there exists a geodesic $p \in \mathcal{G}(f, g)$ that essentially penetrates $x H_{\lambda}$. For technical reasons we will also say $x H_{\lambda}$ is $(f, g)$-separating whenever $f$ and $g$ are both elements of $x H_{\lambda}$ and $f \neq g$; in this case we say $x H_{\lambda}$ is trivially $(f, g)$-separating. The set of all $(f, g)$-separating cosets of $H_{\lambda}$ is denoted by $S_{\lambda}(f, g)$.

The following lemma immediately follows from the definition and the facts that $f, g, h \in G$ and $p \in \mathcal{G}(f, g)$, then $p^{-1} \in \mathcal{G}(g, f)$ and $h p \in \mathcal{G}(h f, h g)$.

Lemma 3.2 For any $f, g, h \in G$ and any $\lambda \in \Lambda$, the following holds:

(a) $S_{\lambda}(f, g)=S_{\lambda}(g, f)$.

(b) $S_{\lambda}(h f, h g)=\left\{h x H_{\lambda} \mid x H_{\lambda} \in S_{\lambda}(f, g)\right\}$.

The terminology in Definition 3.1 is justified by the first claim of following.

Lemma 3.3 For any $\lambda \in \Lambda$, any $f, g \in G$ such that $f^{-1} g \notin H_{\lambda}$, and any $(f, g)-$ separating coset $x H_{\lambda}$, the following hold:

(a) Every path in $\Gamma(G, X \sqcup \mathcal{H})$ connecting $f$ to $g$ and composed of at most 2 geodesics penetrates $x H_{\lambda}$.

(b) For any $p, q \in \mathcal{G}(f, g)$, we have

$$
\begin{gathered}
\widehat{\mathrm{d}}_{\lambda}\left(p_{\text {in }}\left(x H_{\lambda}\right), q_{\text {in }}\left(x H_{\lambda}\right)\right) \leq 3 C, \\
\widehat{\mathrm{d}}_{\lambda}\left(p_{\text {out }}\left(x H_{\lambda}\right), q_{\text {out }}\left(x H_{\lambda}\right)\right) \leq 3 C .
\end{gathered}
$$

Proof Let $x H_{\lambda} \in S_{\lambda}(f, g)$ be $(f, g)$-separating coset. Since $f^{-1} g \notin H_{\lambda}, x H_{\lambda}$ is nontrivially separating. Thus there exists a geodesic $p \in \mathcal{G}(f, g)$ that essentially penetrates $x H_{\lambda}$; let $a$ denote the corresponding $H_{\lambda}$-component of $p$. Let $r$ be any other path in $\Gamma(G, X \sqcup \mathcal{H})$ connecting $f$ to $g$ and composed of at most 2 geodesics. If $a$ is isolated in the loop $p r^{-1}$, we obtain $\widehat{\mathrm{d}}_{\lambda}\left(a_{-}, a_{+}\right) \leq 3 C$ by Lemma 2.4. This contradicts the assumption that $p$ essentially penetrates $x H_{\lambda}$. Hence $a$ is not isolated in $p r^{-1}$. Since $p$ is geodesic, $a$ cannot be connected to a component of $p$. Therefore $a$ is connected to a component of $r$, ie $r$ penetrates $x H_{\lambda}$.

Further let $p, q \in \mathcal{G}(a, b)$ and $x H_{\lambda} \in S_{\lambda}(f, g)$. By part (a) we have $p=p_{1} a p_{2}$ and $q=q_{1} b q_{2}$, where $\left(p_{1}\right)_{+} \in x H_{\lambda},\left(q_{1}\right)_{+} \in x H_{\lambda}$ and $a, b$ are $H_{\lambda}$-components of $p$ and $q$, respectively (see Figure 2). (Of course, $p_{i}$ or $q_{i}, i=1,2$, can be trivial). Then $a$ and $b$ are connected. Let $e$ be an edge or the trivial path connecting $a_{-}$to $b_{-}$ and labeled by a letter from $H_{\lambda} \backslash\{1\}$. Applying Lemma 2.4 to the geodesic triangle $p_{1} e q_{1}^{-1}$, we obtain $\widehat{\mathrm{d}}_{\lambda}\left(e_{-}, e_{+}\right) \leq 3 C$, which gives us the first inequality in (b). The proof of the second inequality is symmetric. 
Corollary 3.4 For any $f, g \in G$ and any $\lambda \in \Lambda$, we have $\left|S_{\lambda}(f, g)\right| \leq \mathrm{d}_{X \cup \mathcal{H}}(f, g)$. In particular, $S_{\lambda}(f, g)$ is finite.

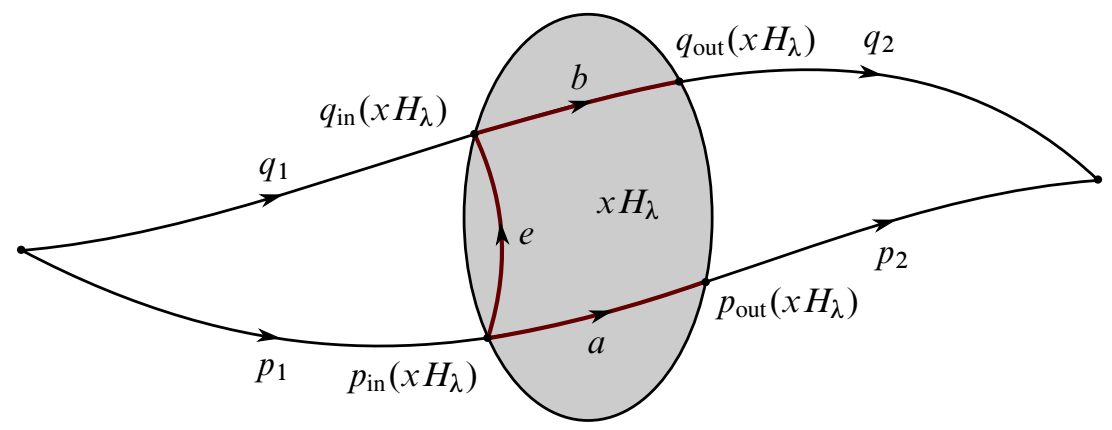

Figure 2

In this section we will use the following elementary observation several times.

Lemma 3.5 Let $p$ be a geodesic in $\Gamma(G, X \sqcup \mathcal{H})$. Suppose that $p$ penetrates a coset $x H_{\lambda}$. Let $p_{0}$ be the initial subpath of $p$ ending at $p_{\text {in }}\left(x H_{\lambda}\right)$. Then $\ell\left(p_{0}\right)=$ $\mathrm{d}_{X \cup \mathcal{H}}\left(p_{-}, x H_{\lambda}\right)$.

Proof Clearly $\mathrm{d}_{X \cup \mathcal{H}}\left(p_{-}, x H_{\lambda}\right) \leq \ell\left(p_{0}\right)$. Suppose that $\mathrm{d}_{X \cup \mathcal{H}}\left(p_{-}, x H_{\lambda}\right)<\ell\left(p_{0}\right)$. Since $x H_{\lambda}$ has diameter 1 with respect to the metric $\mathrm{d}_{X \cup \mathcal{H}}$, we obtain

$$
\mathrm{d}_{X \cup \mathcal{H}}\left(p_{-}, p_{\text {out }}\left(x H_{\lambda}\right)\right) \leq \mathrm{d}_{X \cup \mathcal{H}}\left(p_{-}, x H_{\lambda}\right)+1<\ell\left(p_{0}\right)+1 .
$$

However we obviously have $\ell\left(p_{0}\right)+1=\mathrm{d}_{X \cup \mathcal{H}}\left(p_{-}, p_{\text {out }}\left(x H_{\lambda}\right)\right)$. A contradiction.

Definition 3.6 Given any $f, g \in G$, we define a relation $\preceq$ on the set $S_{\lambda}(f, g)$ as follows:

$$
x H_{\lambda} \preceq y H_{\lambda} \text { iff } \mathrm{d}_{X \cup \mathcal{H}}\left(f, x H_{\lambda}\right) \leq \mathrm{d}_{X \cup \mathcal{H}}\left(f, y H_{\lambda}\right) .
$$

The next lemma is an immediate consequence of Lemma 3.3 and Lemma 3.5.

Lemma 3.7 For any $f, g \in G$ and any $\lambda \in \Lambda$ with $f^{-1} g \notin H_{\lambda}$, $\preceq$ is a linear order on $S_{\lambda}(f, g)$ and every geodesic $p \in \mathcal{G}(f, g)$ penetrates all $(f, g)$-separating cosets according to the order $\preceq$. That is, $S_{\lambda}(f, g)=\left\{x_{1} H_{\lambda} \preceq x_{2} H_{\lambda} \preceq \cdots \preceq x_{n} H_{\lambda}\right\}$ for some $n \in \mathbb{N}$ and $p$ decomposes as

$$
p=p_{1} a_{1} \cdots p_{n} a_{n} p_{n+1},
$$

where $a_{i}$ is an $H_{\lambda}$-component of $p$ and $\left(p_{i}\right)_{+} \in x_{i} H_{\lambda}$ for $i=1, \ldots, n$ (see Figure 3). 


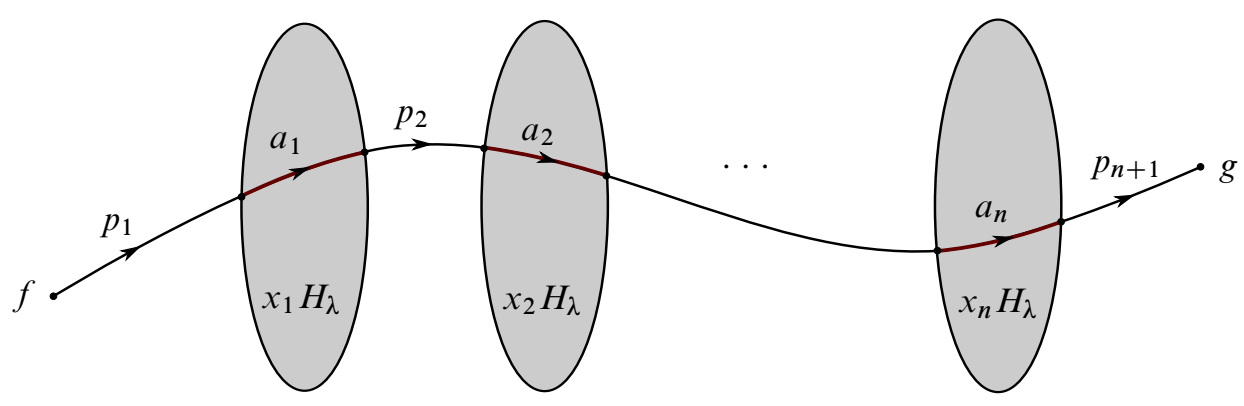

Figure 3

Given $f, g \in G$ and $x H_{\lambda} \in S_{\lambda}(f, g)$, we denote by $E\left(f, g ; x H_{\lambda}\right)$ the set of ordered pairs of entrance-exit points of geodesics from $\mathcal{G}(f, g)$ in the coset $x H_{\lambda}$. That is,

$$
E\left(f, g ; x H_{\lambda}\right)=\left\{\left(p_{\text {in }}\left(x H_{\lambda}\right), p_{\text {out }}\left(x H_{\lambda}\right)\right) \mid p \in \mathcal{G}(f, g)\right\} .
$$

Lemma 3.8 For any $\lambda \in \Lambda$ and any $f, g, h, x \in G$, the following hold:

(a) $E\left(g, f ; x H_{\lambda}\right)=\left\{(v, u) \mid(u, v) \in E\left(f, g ; x H_{\lambda}\right)\right\}$.

(b) $E\left(h f, h g ; x H_{\lambda}\right)=\left\{(h u, h v) \mid(u, v) \in E\left(f, g ; x H_{\lambda}\right)\right\}$.

(c) $\left|E\left(f, g ; x H_{\lambda}\right)\right|<\infty$.

Proof Parts (a) and (b) follow immediately from Lemma 3.2. To prove (c), note that if $x H_{\lambda}$ trivially separates $f$ and $g$, then $E\left(f, g ; x H_{\lambda}\right)=\{(f, g)\}$. Further if $x H_{\lambda}$ separates $f$ and $g$ nontrivially, fix any $(u, v) \in E\left(f, g ; x H_{\lambda}\right)$. Then for any other $\left(u^{\prime}, v^{\prime}\right) \in E\left(f, g ; x H_{\lambda}\right)$, we have $\widehat{\mathrm{d}}_{\lambda}\left(u, u^{\prime}\right)<3 C$ and $\widehat{\mathrm{d}}_{\lambda}\left(v, v^{\prime}\right)<3 C$ by part (b) of Lemma 3.3. Recall that $\left(H_{\lambda}, \widehat{\mathrm{d}}_{\lambda}\right)$ is a locally finite metric space by the definition of a hyperbolically embedded collection of subgroups. Hence $\left|E\left(f, g ; x H_{\lambda}\right)\right|<\infty$.

The main result of this section is the following.

Lemma 3.9 For any $f, g, h \in G$ and any $\lambda \in \Lambda$, the set of all $(f, g)$-separating cosets of $H_{\lambda}$ can be decomposed as

$$
S_{\lambda}(f, g)=S^{\prime} \sqcup S^{\prime \prime} \sqcup F,
$$

where:

(a) $S^{\prime} \subseteq S_{\lambda}(f, h) \backslash S_{\lambda}(h, g)$ and for every $x H_{\lambda} \in S^{\prime}$ we have $E\left(f, g ; x H_{\lambda}\right)=$ $E\left(f, h ; x H_{\lambda}\right)$.

(b) $S^{\prime \prime} \subseteq S_{\lambda}(h, g) \backslash S_{\lambda}(f, h)$ and for every $x H_{\lambda} \in S^{\prime \prime}$ we have $E\left(f, g ; x H_{\lambda}\right)=$ $E\left(h, g ; x H_{\lambda}\right)$.

(c) $|F| \leq 2$. 
Proof First, if $\left|S_{\lambda}(f, g)\right| \leq 2$ the statement is trivial, so we can assume $\left|S_{\lambda}(f, g)\right|>2$. Let

$$
S_{\lambda}(f, g)=\left\{x_{1} H_{\lambda} \preceq x_{2} H_{\lambda} \preceq \cdots \preceq x_{n} H_{\lambda}\right\} .
$$

We fix any geodesics $q \in \mathcal{G}(h, g)$ and $r \in \mathcal{G}(f, h)$. By the first claim of Lemma 3.3, every coset from $S_{\lambda}(f, g)$ is penetrated by at least one of $q, r$. Without loss of generality we may assume that at least one of the cosets from $S_{\lambda}(f, g)$ is penetrated by $r$. Let $x_{i} H$ be the largest coset (with respect to the order $\preceq$ ) that is penetrated by $r$. Thus if $i<n$, then $x_{i+1} H$ is penetrated by $q$. Let

$$
\begin{gathered}
S^{\prime}=\left\{x_{j} H_{\lambda} \mid 1 \leq j<i\right\}, \\
S^{\prime \prime}=\left\{x_{j} H_{\lambda} \mid i+1<j \leq n\right\}, \\
F=S_{\lambda}(f, g) \backslash\left(S^{\prime} \cup S^{\prime \prime}\right) .
\end{gathered}
$$

Obviously $|F| \leq 2$. It remains to prove (a) and (b). We will prove (a) only, the proof of (b) is symmetric.

Fix any $1 \leq j<i$. Let $p$ be any geodesic from $\mathcal{G}(f, g)$. By Lemma 3.7, $p$ decomposes as

$$
p=p_{1} a_{1} p_{2} a_{2} p_{3},
$$

where $a_{1}, a_{2}$ are $H_{\lambda}$-components of $p,\left(p_{1}\right)_{+} \in x_{j} H_{\lambda}$, and $\left(p_{2}\right)_{+} \in x_{i} H_{\lambda}$. Similarly by the choice of $i, r$ decomposes as

$$
r=r_{1} b r_{2}
$$

where $b$ is an $H_{\lambda}$-component of $r$ and $\left(r_{2}\right)_{-} \in x_{i} H_{\lambda}$ (see Figure 4).

Since $\left(r_{2}\right)_{-}$and $\left(p_{2}\right)_{+}$belong to the same coset of $H_{\lambda}$, there exists a path $e$ in $\Gamma(G, X \sqcup \mathcal{H})$ of length at most 1 such that $e_{-}=\left(p_{2}\right)_{+}$and $e_{+}=\left(r_{2}\right)_{-}$. By Lemma 3.5, we have $\ell\left(p_{1} a_{1} p_{2}\right)=\ell\left(r_{1}\right)$. Hence the path $t=p_{1} a_{1} p_{2} e r_{2}$ has the same length as $r$, ie $t \in \mathcal{G}(f, h)$. Also,

$$
\begin{gathered}
p_{\text {in }}\left(x_{j} H_{\lambda}\right)=t_{\text {in }}\left(x_{j} H_{\lambda}\right), \\
p_{\text {out }}\left(x_{j} H_{\lambda}\right)=t_{\text {out }}\left(x_{j} H_{\lambda}\right) .
\end{gathered}
$$

So far all our arguments were valid for any $p \in \mathcal{G}(f, g)$. Since $x_{j} H_{\lambda} \in S_{\lambda}(f, g)$, there exists $p \in \mathcal{G}(f, g)$ that essentially penetrates $x_{j} H_{\lambda}$, ie $\widehat{\mathrm{d}}_{\lambda}\left(\left(a_{1}\right)_{-},\left(a_{1}\right)_{+}\right)>3 C$ in the above notation. In this case $t$ also essentially penetrates $x_{j} H_{\lambda}$. Thus $x_{j} H \in S_{\lambda}(f, h)$. Moreover since we have (4) and (5) for every $p \in \mathcal{G}(f, g)$, we obtain $E\left(f, g ; x_{j} H_{\lambda}\right)=$ $E\left(f, h ; x_{j} H_{\lambda}\right)$. 


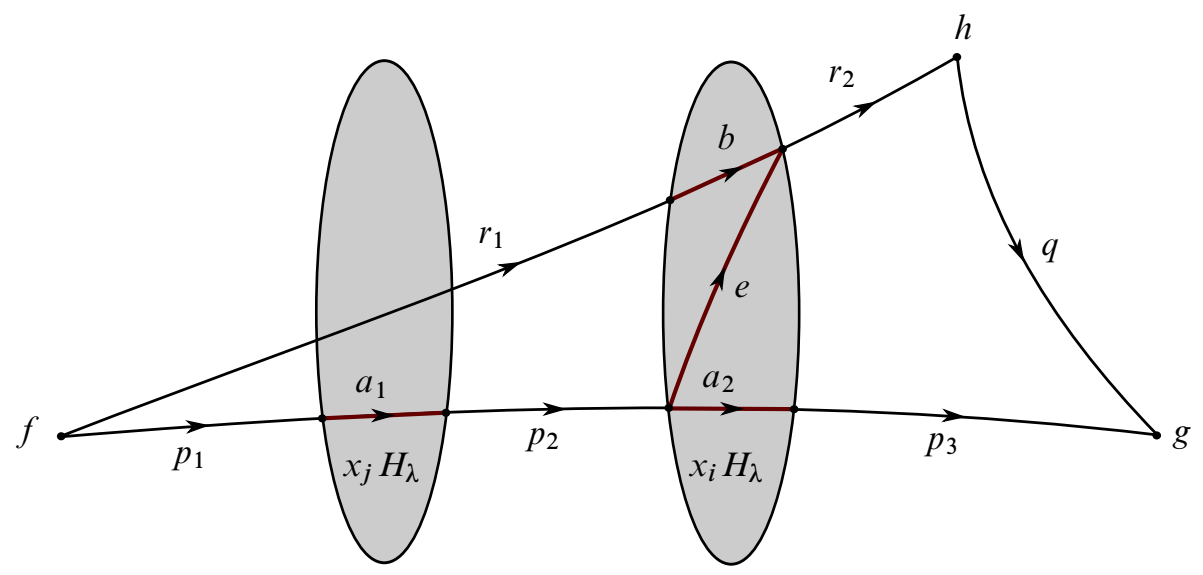

Figure 4

To complete the proof of (a) it remains to show that $x_{j} H_{\lambda} \notin S_{\lambda}(h, g)$. Clearly $g \notin x_{j} H_{\lambda}$, or $p$ would not be geodesic, so $x_{j} H_{\lambda}$ does not trivially separate $g$ and $h$. Thus, if $x_{j} H_{\lambda} \in S_{\lambda}(h, g)$ there must be a geodesic from $h$ to $g$ which essentially penetrates $x_{j} H_{\lambda}$. Hence by Lemma 3.3, every geodesic from $h$ to $g$ penetrates $x_{j} H_{\lambda}$, which means $q$ penetrates $x_{j} H_{\lambda}$. Then using Lemma 3.5, the fact that every coset of $H_{\lambda}$ has diameter 1 with respect to the metric $\mathrm{d}_{X \cup \mathcal{H}}$, and the triangle inequality, we obtain

$$
\begin{aligned}
\ell(q) & =\mathrm{d}_{X \cup \mathcal{H}}\left(h, x_{j} H_{\lambda}\right)+1+\mathrm{d}_{X \cup \mathcal{H}}\left(g, x_{j} H_{\lambda}\right) \\
& >\mathrm{d}_{X \cup \mathcal{H}}\left(h, x_{i} H_{\lambda}\right)+1+\mathrm{d}_{X \cup \mathcal{H}}\left(g, x_{i} H_{\lambda}\right) \\
& \geq \ell\left(r_{2}\right)+\mathrm{d}_{X \cup \mathcal{H}}\left(\left(r_{2}\right)_{-},\left(p_{3}\right)_{-}\right)+\ell\left(p_{3}\right) \\
& \geq \mathrm{d}_{X \cup \mathcal{H}}(h, g) .
\end{aligned}
$$

Since one of the inequalities is strict, this contradicts the assumption that $q$ is geodesic.

\section{Extending quasicocycles}

We keep all assumptions and notation from the previous section. For each $\lambda \in \Lambda$, let

$$
\mathcal{F}_{\lambda}=\left\{h \in H_{\lambda} \mid h \in H_{\mu} \text { for some } \mu \neq \lambda\right\} .
$$

In particular, if $\left\{H_{\lambda}\right\}_{\lambda \in \Lambda}$ consists of a single subgroup $H$, the corresponding subset $\mathcal{F}=\varnothing$.

It follows from Lemma 2.4 that every $h \in F_{\lambda}$ satisfies $\widehat{\mathrm{d}}_{\lambda}(1, h) \leq 2 C$, where $C$ is the constant from Lemma 2.4. Indeed for every such $h$ there is a loop $e_{1} e_{2}$ in $\Gamma(G, X \sqcup \mathcal{H})$, 
where $e_{1}$ is an edge labeled by $h \in H_{\lambda}$ and $e_{2}$ is an edge labeled by the copy of $h$ in $H_{\mu}$ for some $\mu \in \Lambda$. Since the metric space $\left(H_{\lambda}, \widehat{\mathrm{d}}_{\lambda}\right)$ is locally finite by the definition of a hyperbolically embedded collection of subgroups, we obtain the following.

Lemma 4.1 $\left|\mathcal{F}_{\lambda}\right|<\infty$ for all $\lambda \in \Lambda$.

Also, for $q_{\lambda} \in Q Z^{1}\left(H_{\lambda}, U_{\lambda}\right)$, let

$$
K_{\lambda}=\max \left\{\left\|q_{\lambda}(g)\right\|: \widehat{\mathrm{d}}_{\lambda}(1, g)<15 C\right\} .
$$

Observe that the constant $K_{\lambda}$ is well-defined by local finiteness of $\left(H_{\lambda}, \widehat{\mathrm{d}}_{\lambda}\right)$.

We can now state our main extension theorem in its full generality. Recall that for a quasicocycle $q, D(q)$ denotes its defect defined by (2).

Theorem 4.2 Let $G$ be a group, $\left\{H_{\lambda}\right\}_{\lambda \in \Lambda}$ a hyperbolically embedded collection of subgroups of $G, V$ a normed $G$-module. For each $\lambda \in \Lambda$, let $U_{\lambda}$ be an $H_{\lambda}$-submodule of $G$. Then there exists a linear map

$$
\iota: \bigoplus_{\lambda \in \Lambda} Q Z_{\text {as }}^{1}\left(H_{\lambda}, U_{\lambda}\right) \rightarrow Q Z_{\text {as }}^{1}(G, V)
$$

such that for any $q=\left(q_{\lambda}\right)_{\lambda \in \Lambda} \in \bigoplus_{\lambda \in \Lambda} Q Z_{\text {as }}^{1}\left(H_{\lambda}, U_{\lambda}\right)$ the following hold:

(a) For any $\lambda \in \Lambda$ and any $h \in H_{\lambda} \backslash \mathcal{F}_{\lambda}$, we have $\iota(q)(h)=q_{\lambda}(h)$. In particular,

$$
\sup _{h \in H_{\lambda}}\left\|\iota(q)(h)-q_{\lambda}(h)\right\|<\infty .
$$

(b) $D(\iota(q)) \leq \sum_{\lambda}\left(54 K_{\lambda}+66 D\left(q_{\lambda}\right)\right)$.

Notice that the sum in part (b) is finite because $q_{\lambda} \equiv 0$ for all but finitely many $\lambda$, and thus $K_{\lambda}=D\left(q_{\lambda}\right)=0$ for all but finitely many $\lambda$. If $G$ contains a singe hyperbolically embedded subgroup, Theorem 4.2 obviously reduces to Theorem 1.4 mentioned in the introduction. Using Lemma 2.5, one can also obtain a general version of Corollary 1.7. We leave this to the reader.

Throughout the rest of the section, we use the notation of Theorem 4.2. Although our proof can be entirely written in the language of quasicocycles, the following concept helps making some arguments more symmetric and easier to comprehend. In the definition below, we write $s(a)=t(a)$ for two partial maps $s, t: A \rightarrow B$ if the value $s(a)$ is defined if and only if $t(a)$ is, and these values are equal whenever defined. 
Definition 4.3 A partial bicombing of $G$ with coefficients in $V$ is a partial map $r: G \times G \rightarrow V$. We say that

(a) $r$ is $G$-equivariant if $h r(f, g)=r(h f, h g)$ for any $f, g, h \in G$.

(b) $r$ is antisymmetric if $r(f, g)=-r(g, f)$ for any $f, g \in G$.

(c) $r$ has bounded area if there exists a constant $A$ such that for any $f, g, h \in G$ for which $r(f, g), r(g, h)$, and $r(h, f)$ are defined, we have

$$
\|r(f, g)+r(g, h)+r(h, f)\| \leq A .
$$

The infimum of all $A$ satisfying (7) is called the area of $r$ and is denoted by $A(r)$.

Let us fix $\lambda \in \Lambda$. Given $q_{\lambda} \in Q Z_{a s}^{1}\left(H_{\lambda}, U_{\lambda}\right)$, we define a partial map $r_{\lambda}: G \times G \rightarrow V$ by

$$
r_{\lambda}(f, g)=f q_{\lambda}\left(f^{-1} g\right) .
$$

Thus $r_{\lambda}(f, g)$ is defined if and only if $f$ and $g$ belong to the same coset $x H_{\lambda}$.

Lemma 4.4 The partial map $r_{\lambda}: G \times G \rightarrow V$ is an antisymmetric equivariant partial bicombing of $G$ of area

$$
A\left(r_{\lambda}\right) \leq D\left(q_{\lambda}\right) .
$$

Proof Equivariance of $r_{\lambda}$ is obvious and antisymmetry follows immediately from antisymmetry of $q_{\lambda}$. By equivariance it suffices to verify the bounded area condition for the a triple $1, g, h \in G$. We have

$$
\left\|r_{\lambda}(1, g)+r_{\lambda}(g, h)+r_{\lambda}(h, 1)\right\|=\left\|q_{\lambda}(g)+g q_{\lambda}\left(g^{-1} h\right)-q_{\lambda}(h)\right\| \leq D\left(q_{\lambda}\right) .
$$

Corollary 4.5 For any $n \in \mathbb{N}$, any $x \in G$, and any $g_{0}, \ldots, g_{n} \in x H_{\lambda}$, we have

$$
\left\|r_{\lambda}\left(g_{0}, g_{n}\right)-\sum_{i=1}^{n} r_{\lambda}\left(g_{i-1}, g_{i}\right)\right\| \leq(n-1) D\left(q_{\lambda}\right) \text {. }
$$

Proof The claim follows from antisymmetry, the definition of area, and (8) by induction.

Our next goal is to construct a globally defined antisymmetric bounded area $G-$ equivariant bicombing $\widetilde{r}_{\lambda}: G \times G \rightarrow V$ that extends $r_{\lambda}$. To this end, for each $f, g \in G$ and each coset $x H_{\lambda}$, we define the average

$$
R_{\mathrm{av}}\left(f, g ; x H_{\lambda}\right)=\frac{1}{\left|E\left(f, g ; x H_{\lambda}\right)\right|} \sum_{\substack{(u, v) \in \\ E\left(f, g ; x H_{\lambda}\right)}} r_{\lambda}(u, v) .
$$


If $x H_{\lambda} \notin S_{\lambda}(f, g)$, we assume $R_{\mathrm{av}}\left(f, g ; x H_{\lambda}\right)=0$. Note that $R_{\mathrm{av}}\left(f, g ; x H_{\lambda}\right)$ is well-defined since $E\left(f, g ; x H_{\lambda}\right)<\infty$ by part (c) of Lemma 3.8.

Lemma 4.6 For any $f, g, h, x \in G$, the following hold:

(a) $R_{\mathrm{av}}\left(f, g ; x H_{\lambda}\right)=-R_{\mathrm{av}}\left(g, f ; x H_{\lambda}\right)$.

(b) $R_{\mathrm{av}}\left(h f, h g ; h x H_{\lambda}\right)=R_{\mathrm{av}}\left(f, g ; x H_{\lambda}\right)$.

(c) For any $(u, v) \in E\left(f, g ; x H_{\lambda}\right)$, we have

$$
\left\|r_{\lambda}(u, v)-R_{\mathrm{av}}\left(f, g ; x H_{\lambda}\right)\right\| \leq 2 D\left(q_{\lambda}\right)+2 K_{\lambda} .
$$

Proof The first claim follows from parts (a) of Lemma 3.8 and antisymmetry of $r_{\lambda}$. The second claim follows from parts (b) of Lemma 3.8 and the equivariance of $r_{\lambda}$.

To prove (c), note that for any $\left(u^{\prime}, v^{\prime}\right) \in E\left(f, g ; x H_{\lambda}\right)$, we have

$$
\max \left\{\widehat{\mathrm{d}}_{\lambda}\left(u, u^{\prime}\right), \widehat{\mathrm{d}}_{\lambda}\left(v, v^{\prime}\right)\right\} \leq 3 C
$$

by Lemma 3.3. Thus, using the triangle inequality and applying Corollary 4.5 to elements $u, u^{\prime}, v^{\prime}, v \in x H_{\lambda}$, we obtain

$$
\begin{aligned}
\left\|r_{\lambda}(u, v)-r_{\lambda}\left(u^{\prime}, v^{\prime}\right)\right\| & \leq\left\|r_{\lambda}(u, v)-r_{\lambda}\left(u, u^{\prime}\right)-r_{\lambda}\left(u^{\prime}, v^{\prime}\right)-r_{\lambda}\left(v^{\prime}, v\right)\right\| \\
& +\left\|r_{\lambda}\left(u, u^{\prime}\right)\right\|+\left\|r_{\lambda}\left(v^{\prime}, v\right)\right\| \\
& \leq 2 D\left(q_{\lambda}\right)+2 K_{\lambda} .
\end{aligned}
$$

This obviously implies (9).

Let

$$
\widetilde{r}_{\lambda}(f, g)=\sum_{x H_{\lambda} \in S_{\lambda}(f, g)} R_{\mathrm{av}}\left(f, g ; x H_{\lambda}\right) .
$$

Note that $\widetilde{r}_{\lambda}$ is well-defined as $S_{\lambda}(f, g)$ is finite for any $f, g \in G$ by Corollary 3.4.

Lemma 4.7 The map $\widetilde{r}_{\lambda}: G \times G \rightarrow V$ is an antisymmetric $G$-equivariant bicombing of area

$$
A\left(\widetilde{r}_{\lambda}\right) \leq 66 D\left(q_{\lambda}\right)+54 K_{\lambda} .
$$

Proof Equivariance and antisymmetry of $\widetilde{r}_{\lambda}$ follow immediately from Lemma 3.2 and Lemma 4.6. In order to show that $\widetilde{r}_{\lambda}$ satisfies the bounded area condition, we need to estimate the norm of $\widetilde{r}_{\lambda}(f, g)+\widetilde{r}_{\lambda}(g, h)+\widetilde{r}_{\lambda}(h, f)$ uniformly on $f, g, h \in G$. Since $R_{\mathrm{av}}\left(f, g ; x H_{\lambda}\right)=0$ if $x H_{\lambda} \notin S_{\lambda}(f, g)$, we have

$$
\widetilde{r}_{\lambda}(f, g)+\widetilde{r}_{\lambda}(g, h)+\widetilde{r}_{\lambda}(h, f)=\sum_{x H_{\lambda} \in G / H_{\lambda}} \rho\left(f, g, h ; x H_{\lambda}\right),
$$


where

$$
\rho\left(f, g, h ; x H_{\lambda}\right):=R_{\mathrm{av}}\left(f, g ; x H_{\lambda}\right)+R_{\mathrm{av}}\left(g, h ; x H_{\lambda}\right)+R_{\mathrm{av}}\left(h, f ; x H_{\lambda}\right) .
$$

Of course, $\rho\left(f, g, h ; x H_{\lambda}\right)$ is nontrivial only if $x H_{\lambda} \in S_{\lambda}(f, g) \cup S_{\lambda}(g, h) \cup S_{\lambda}(h, f)$.

Fix $f, g, h \in G$. We start by estimating $\rho\left(f, g, h ; x H_{\lambda}\right)$ for cosets from $S_{\lambda}(f, g)$. Let $S_{\lambda}(f, g)=S^{\prime} \sqcup S^{\prime \prime} \sqcup F$ be the decomposition provided by Lemma 3.9. Suppose first that $x H_{\lambda} \in S^{\prime}$. Then $x H_{\lambda} \in S_{\lambda}(f, h)=S_{\lambda}(h, f)$ and $E\left(f, g ; x H_{\lambda}\right)=E\left(f, h ; x H_{\lambda}\right)$ by Lemma 3.9. Hence

$$
R_{\mathrm{av}}\left(f, g ; x H_{\lambda}\right)=R_{\mathrm{av}}\left(f, h ; x H_{\lambda}\right)=-R_{\mathrm{av}}\left(h, f ; x H_{\lambda}\right) .
$$

by Lemma 4.6 (a). On the other hand, Lemma 3.9 also states that $x H_{\lambda} \notin S_{\lambda}(h, g)=$ $S_{\lambda}(g, h)$. Hence

$$
R_{\mathrm{av}}\left(g, h ; x H_{\lambda}\right)=0 .
$$

Summing up (11) and (12), we obtain $\rho\left(f, g, h ; x H_{\lambda}\right)=0$. Similarly,

$$
\rho\left(f, g, h ; x H_{\lambda}\right)=0
$$

for any $x H_{\lambda} \in S^{\prime \prime}$. Thus

$$
\sum_{\substack{x H_{\lambda} \in \\ S_{\lambda}(f, g)}} \rho\left(f, g, h ; x H_{\lambda}\right)=\sum_{x H_{\lambda} \in F} \rho\left(f, g, h ; x H_{\lambda}\right) .
$$

Fix a coset $x H_{\lambda} \in F$ and any $p \in \mathcal{G}(f, g), q \in \mathcal{G}(h, g), r \in \mathcal{G}(f, h)$. There are three cases to consider.

Case $1 x H_{\lambda} \in S_{\lambda}(g, h) \cap S_{\lambda}(h, f)$. In this case we have $p=p_{1} a p_{2}, q=q_{1} c q_{2}$, $r=r_{1} b r_{2}$, where $a, c$ and $b$ are $H_{\lambda}$-components of $p, q$ and $r$, respectively, corresponding to the coset $x H_{\lambda}$ (ie $a_{ \pm}, b_{ \pm}, c_{ \pm} \in x H_{\lambda}$ ). Let $e_{1}, e_{2}, e_{3}$ be paths of lengths at most 1 labeled by elements of $H_{\lambda}$ and connecting $a_{-}$to $b_{-}, b_{+}$to $c_{-}$, and $c_{+}$to $a_{+}$(see Figure 5).

Since a geodesic in $\Gamma(G, X \sqcup \mathcal{H})$ can penetrate a coset of $H_{\lambda}$ at most once, $e_{1}$ is either trivial or is an isolated component of a geodesic triangle (namely $p_{1} e_{1} r_{1}^{-1}$ ). The same holds true for $e_{1}$ and $e_{2}$. Hence by Lemma 2.4, we obtain

$$
\widehat{\mathrm{d}}_{\lambda}\left(\left(e_{i}\right)_{-},\left(e_{i}\right)_{+}\right) \leq 3 C, \quad i=1,2,3 \text {. }
$$

In particular,

$$
\left\|r_{\lambda}\left(\left(e_{i}\right)_{-},\left(e_{i}\right)_{+}\right)\right\| \leq K_{\lambda}, \quad i=1,2,3 .
$$




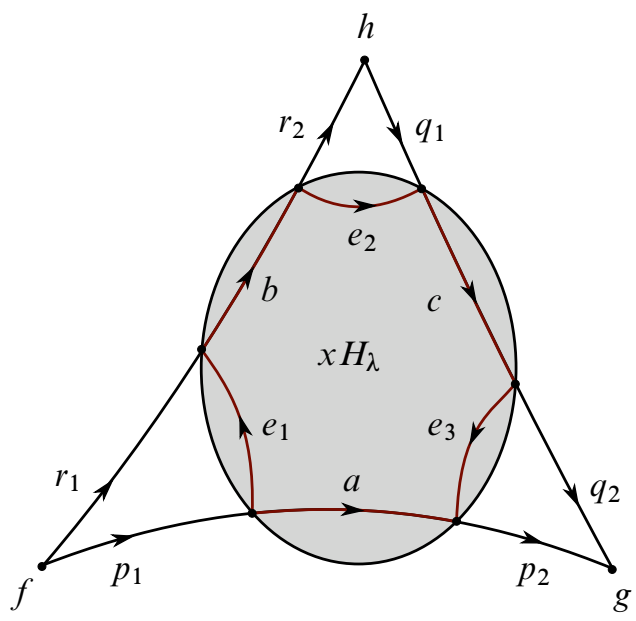

Figure 5

by the definition of $K_{\lambda}$ (see (6)). Using the triangle inequality, applying Corollary 4.5 to the vertices of the hexagon $e_{1} b e_{2} c e_{3} a^{-1}$, and using (15), we obtain

$\left\|r_{\lambda}\left(a_{-}, a_{+}\right)+r_{\lambda}\left(b_{+}, b_{-}\right)+r_{\lambda}\left(c_{+}, c_{-}\right)\right\|$

$$
\begin{aligned}
\leq \| r_{\lambda}\left(a_{-}, a_{+}\right)-r_{\lambda}\left(b_{-}, b_{+}\right)-r_{\lambda}\left(c_{-}, c_{+}\right) & -\sum_{i=1}^{3} r_{\lambda}\left(\left(e_{i}\right)_{-},\left(e_{i}\right)_{+}\right) \| \\
& +\left\|\sum_{i=1}^{3} r_{\lambda}\left(\left(e_{i}\right)_{-},\left(e_{i}\right)_{+}\right)\right\| \\
& \leq 5 D\left(q_{\lambda}\right)+3 K_{\lambda} .
\end{aligned}
$$

Now Lemma 4.6 (c) implies

(16) $\left\|\rho\left(f, g, h ; x H_{\lambda}\right)\right\|$

$$
\begin{aligned}
& =\left\|R_{\mathrm{av}}\left(f, g ; x H_{\lambda}\right)+R_{\mathrm{av}}\left(g, h ; x H_{\lambda}\right)+R_{\mathrm{av}}\left(h, f ; x H_{\lambda}\right)\right\| \\
& \leq\left\|r_{\lambda}\left(a_{-}, a_{+}\right)+r_{\lambda}\left(c_{+}, c_{-}\right)+r_{\lambda}\left(b_{+}, b_{-}\right)\right\|+6\left(D\left(q_{\lambda}\right)+K_{\lambda}\right) \\
& \leq 11 D\left(q_{\lambda}\right)+9 K_{\lambda} .
\end{aligned}
$$

Case $2 x H_{\lambda} \in S_{\lambda}(h, f) \backslash S_{\lambda}(g, h)$ or $x H_{\lambda} \in S_{\lambda}(g, h) \backslash S_{\lambda}(h, f)$. Since the proof in these cases is the same, we will only consider the case $x H_{\lambda} \in S_{\lambda}(h, f) \backslash S_{\lambda}(g, h)$. Let $p=p_{1} a p_{2}, r=r_{1} b r_{2}$, and $e_{1}$ be as in Case 1 and let $e$ be the path of length at most 1 in $\Gamma(G, X \sqcup \mathcal{H})$ connecting $b_{+}$to $a_{+}$and labeled by an element of $H_{\lambda}$. There are two possibilities to consider. 
(2a) First assume that $e$ is isolated in the quadrilateral $e p_{2} q^{-1} r_{2}^{-1}$ (see Figure 6). Then we have $\widehat{\mathrm{d}}_{\lambda}\left(e_{-}, e_{+}\right) \leq 4 C$ by Lemma 2.4 and hence

$$
\left\|r_{\lambda}\left(e_{-}, e_{+}\right)\right\| \leq K_{\lambda} .
$$

Note that (15) remains valid for $i=1$. Applying Corollary 4.5 to the vertices of the quadrilateral $e_{1} b e a^{-1}$ as in Case 1 we obtain

$$
\begin{aligned}
\| r_{\lambda}\left(a_{-}, a_{+}\right)+r_{\lambda}\left(b_{+},\right. & \left.b_{-}\right) \| \\
& \leq\left\|r_{\lambda}\left(a_{-}, a_{+}\right)-r_{\lambda}\left(\left(e_{1}\right)_{-},\left(e_{1}\right)_{+}\right)-r_{\lambda}\left(b_{-}, b_{+}\right)-r_{\lambda}\left(e_{-}, e_{+}\right)\right\| \\
& +\left\|r_{\lambda}\left(\left(e_{1}\right)_{-},\left(e_{1}\right)_{+}\right)\right\|+\left\|r_{\lambda}\left(e_{-}, e_{+}\right)\right\| \\
& \leq 3 D\left(q_{\lambda}\right)+2 K_{\lambda} .
\end{aligned}
$$

Since $x H_{\lambda} \notin S_{\lambda}(g, h)$, we have $R_{\mathrm{av}}\left(g, h ; x H_{\lambda}\right)=0$. Finally Lemma 4.6 (c) implies

$$
\begin{aligned}
\left\|\rho\left(f, g, h ; x H_{\lambda}\right)\right\| & =\left\|R_{\mathrm{av}}\left(f, g ; x H_{\lambda}\right)+R_{\mathrm{av}}\left(h, f ; x H_{\lambda}\right)\right\| \\
& \leq\left\|r_{\lambda}\left(a_{-}, a_{+}\right)+r_{\lambda}\left(b_{+}, b_{-}\right)\right\|+4\left(D\left(q_{\lambda}\right)+K_{\lambda}\right) \\
& \leq 7 D\left(q_{\lambda}\right)+6 K_{\lambda} .
\end{aligned}
$$

(2b) Suppose now that $e$ is not isolated in the quadrilateral $e p_{2} q^{-1} r_{2}^{-1}$. Then $e$ is connected to a component $c$ of $q$. Let $q=q_{1} c q_{2}$ and let $e_{1}$ and $e_{2}$ be as in Case 1 (see Figure 5). Then (15) remains valid. In addition, we have $\widehat{\mathrm{d}}_{\lambda}\left(c_{-}, c_{+}\right) \leq 3 C$ as $x H_{\lambda} \notin$ $S_{\lambda}(g, h)$ and hence $q$ can not essentially penetrate $x H_{\lambda}$. Hence $\left\|r_{\lambda}\left(c_{-}, c_{+}\right)\right\| \leq K_{\lambda}$. The reader can easily verify that arguing as in Case 1 and then as in (17), we can obtain

$$
\left\|r_{\lambda}\left(a_{-}, a_{+}\right)+r_{\lambda}\left(b_{+}, b_{-}\right)\right\| \leq 5 A_{\lambda}+4 K_{\lambda}
$$

and consequently

$$
\left\|\rho\left(f, g, h ; x H_{\lambda}\right)\right\| \leq 9 D\left(q_{\lambda}\right)+8 K_{\lambda} .
$$

Case $3 \quad x H_{\lambda} \notin S_{\lambda}(h, f) \cup S_{\lambda}(g, h)$. Let $p=p_{1} a p_{2}$ be as in Cases 1 and 2. There are three possibilities to consider.

(3a) $a$ is an isolated component of $p q^{-1} r^{-1}$. In this case $\widehat{\mathrm{d}}_{\lambda}\left(a_{-}, a_{+}\right) \leq 3 C$.

(3b) $a$ is connected to a component of exactly one of $q, r$. For definiteness, assume that $a$ is connected to a component $b$ of $r$. Then, in the notation of Case 2 (see Figure 6), $e$ is isolated in $e p_{2} q^{-1} r_{2}^{-1}$ and we have $\widehat{\mathrm{d}}_{\lambda}\left(e_{-}, e_{+}\right) \leq 4 C$ by Lemma 2.4. As in Case 1, we have (14) for $i=1$. Since $x H_{\lambda} \notin S_{\lambda}(h, f), r$ can not essentially 


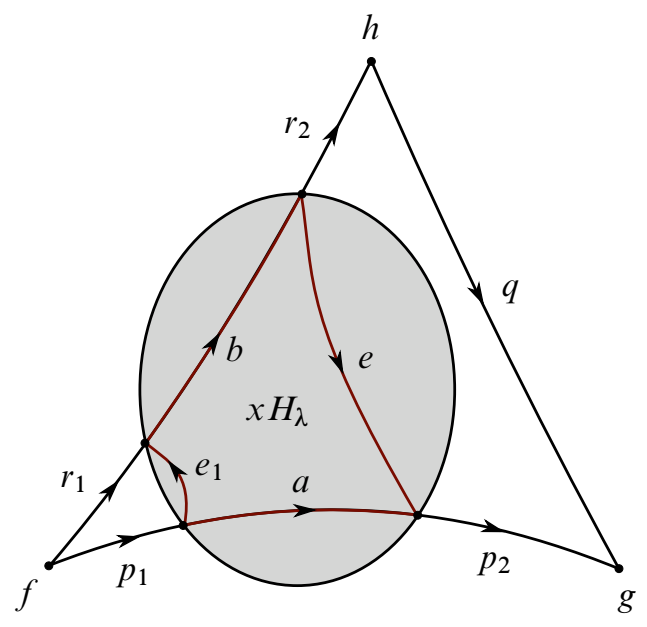

Figure 6

penetrate $x H_{\lambda}$. Thus $\widehat{\mathrm{d}}_{\lambda}\left(b_{-}, b_{+}\right) \leq 3 C$. Applying the triangle inequality to the quadrilateral $e_{1} b e a^{-1}$, we obtain

$$
\widehat{\mathrm{d}}_{\lambda}\left(a_{-}, a_{+}\right) \leq 10 C \text {. }
$$

(3c) $a$ is connected to a component $b$ of $r$ and a component $c$ of $q$. Then in the notation of Case 1 and Figure 5, inequalities (14) remain valid and we also have $\widehat{\mathrm{d}}_{\lambda}\left(b_{-}, b_{+}\right) \leq 3 C$ and $\widehat{\mathrm{d}}_{\lambda}\left(c_{-}, c_{+}\right) \leq 3 C$ as in Case $3 b$. Applying the triangle inequality to the hexagon $e_{1} b e_{2} c e_{3} a^{-1}$, we obtain

$$
\widehat{\mathrm{d}}_{\lambda}\left(a_{-}, a_{+}\right) \leq 15 C \text {. }
$$

Thus, in all Cases 3a)-3c) we have $\left\|r_{\lambda}\left(a_{-}, a_{+}\right)\right\| \leq K_{\lambda}$. Since $R_{\mathrm{av}}\left(g, h ; x H_{\lambda}\right)=$ $R_{\mathrm{av}}\left(h, f ; x H_{\lambda}\right)=0$ in this case, using Lemma 4.6 (c) we obtain

$$
\left\|\rho\left(f, g, h ; x H_{\lambda}\right)\right\|=\left\|R_{\mathrm{av}}\left(f, g ; x H_{\lambda}\right)\right\| \leq 2 A\left(r_{\lambda}\right)+3 K_{\lambda} .
$$

in Case 3.

Summarizing (13), (16), (17), (18), (19), and taking into account that $|F| \leq 2$, we obtain

$$
\left\|\sum_{x H_{\lambda} \in S_{\lambda}(f, g)} \rho\left(f, g, h ; x H_{\lambda}\right)\right\|=\left\|\sum_{x H_{\lambda} \in F} \rho\left(f, g, h ; x H_{\lambda}\right)\right\| \leq 22 D(q \lambda)+18 K_{\lambda} .
$$

Repeating the same arguments for $S_{\lambda}(h, f)$ and $S_{\lambda}(g, f)$ and summing up, we obtain

$$
\left\|\widetilde{r}_{\lambda}(f, g)+\widetilde{r}_{\lambda}(g, h)+\widetilde{r}_{\lambda}(h, f)\right\| \leq 66 D\left(q_{\lambda}\right)+54 K_{\lambda} .
$$


We are now ready to prove the main extension theorem.

Proof of Theorem 4.2 Let $q=\left(q_{\lambda}\right)_{\lambda \in \Lambda} \in \bigoplus_{\lambda \in \Lambda} Q Z_{\text {as }}^{1}\left(H_{\lambda}, U_{\lambda}\right)$. For each $\lambda \in \Lambda$, let $\widetilde{r}_{\lambda}$ be the bicombing constructed above and let $\widetilde{q}_{\lambda}(g)=\widetilde{r}_{\lambda}(1, g)$. Then $\widetilde{q}_{\lambda} \in$ $Q Z_{a s}^{1}(G, V)$. Indeed we have

$$
\begin{aligned}
\left\|\widetilde{q}_{\lambda}(f g)-\widetilde{q}_{\lambda}(f)-f \widetilde{q}_{\lambda}(g)\right\| & =\left\|\widetilde{r}_{\lambda}(1, f g)-\widetilde{r}_{\lambda}(1, f)-f \widetilde{r}_{\lambda}(1, g)\right\| \\
& =\left\|\widetilde{r}_{\lambda}(1, f g)+\widetilde{r}_{\lambda}(f, 1)+\widetilde{r}_{\lambda}(f g, f)\right\| \\
& \leq A\left(\widetilde{r}_{\lambda}\right) .
\end{aligned}
$$

antisymmetry of $\widetilde{q}_{\lambda}$ follows from that of $\widetilde{r}_{\lambda}$.

Further we define

$$
\iota(q)=\sum_{\lambda \in \Lambda} \widetilde{q}_{\lambda}
$$

Since $q$ is supported on only finitely many $\lambda, \iota(q)$ is equal to a finite linear combination of quasicocycles, so $\iota(q) \in Q Z_{a s}^{1}(G, V)$. It is easy to see that the maps $Q Z_{\text {as }}^{1}\left(H_{\lambda}, U_{\lambda}\right) \rightarrow Q Z_{a s}^{1}(G, V)$ defined by $q_{\lambda} \mapsto \widetilde{q}_{\lambda}$ are linear. Hence so is $\iota$.

If $h \in H_{\lambda} \backslash \mathcal{F}_{\lambda}$, then $S_{\lambda}(1, h)=\left\{H_{\lambda}\right\}$ and $S_{\mu}(1, h)=\varnothing$ for any $\mu \neq \lambda$. Obviously $E\left(1, h ; H_{\lambda}\right)=\{(1, h)\}$. Thus $\widetilde{r}_{\lambda}(1, h)=r_{\lambda}(1, h)=q_{\lambda}(h)$ and $\widetilde{r}_{\mu}(1, h)=0$ whenever $\mu \neq \lambda$. Thus

$$
\iota(q)(h)=\sum_{\mu \in \Lambda} \widetilde{q}_{\mu}(h)=\sum_{\mu \in \Lambda} \widetilde{r}_{\mu}(1, h)=q_{\lambda}(h) .
$$

This finishes the proof of (a). Part (b) follows from (20) and (10).

Remark 4.8 Our proof essentially uses the fact that the quasicocycles $q_{\lambda}$ are antisymmetric. In fact, our approach provably fails for nonantisymmetric ones. This can be illustrated in the case when $G=F(x, y)$, the free group of rank 2, and $H=\langle x\rangle$. Indeed take $q \in Q Z^{1}(H, \mathbb{R})$ defined by

$$
q\left(x^{n}\right)= \begin{cases}1 & \text { if } n \geq 0 \\ 0 & \text { if } n<0\end{cases}
$$

Let $\widetilde{q}$ be the extension obtained as above using the subset $X=\{x, y\}$ of $G$. Take any $n \in \mathbb{N}$ such that $\widehat{d}\left(1, x^{n}\right)>3 C$ (in fact, $C=0$ in this case, but we will not use this). Then it is straightforward to verify that $\widetilde{q}\left(\left(y x^{n}\right)^{k}\right)=k$ while $\widetilde{q}\left(\left(y x^{n}\right)^{-k}\right)=$ $\widetilde{q}\left(\left(x^{-n} y^{-1}\right)^{k}\right)=0$ for every $k \in \mathbb{N}$. This contradicts the quasicocycle identity as $k \rightarrow \infty$. A similar argument shows that the antisymmetry condition can not be dropped in Example 1.2. 


\section{Applications}

Bounded cohomology Our goal here is to prove Corollary 1.7. We begin with an auxiliary result.

Proposition 5.1 Let $G$ be a group, $H$ a hyperbolically embedded subgroup of $G, V$ a $G$-module, and $U$ an $H$-submodule of $V$. Suppose that there exists a continuous projection $\pi: V \rightarrow U$. Then there is a linear map $\phi: Q Z^{1}(H, U) \rightarrow E H_{b}^{2}(G, V)$ such that $\operatorname{Ker} \phi \subseteq \ell^{\infty}(H, U)+Z^{1}(H, U)$. In particular,

$$
\operatorname{dim} H_{b}^{2}(G, V) \geq \operatorname{dim} E H_{b}^{2}(G, V) \geq \operatorname{dim} E H_{b}^{2}(H, U) .
$$

Proof We define $\phi$ to be the composition $\delta \circ \varkappa$, where $\varkappa$ is given by Corollary 1.5 and $\delta$ is the natural map $Q Z^{1}(G, V) \rightarrow E H_{b}^{2}(G, V)$ (see Lemma 2.6). Note that if $\phi(q)=0$ for some $q \in Q Z^{1}(H, U)$, then

$$
\varkappa(q)=h+b,
$$

where $b \in \ell^{\infty}(G, V)$ and $h \in Z^{1}(G, V)$. Since $\varkappa(q)(x) \in U$ for all $x \in H$, composing both sides of this equality with $\pi$ and restricting to $H$ we obtain

$$
\left.\varkappa(q)\right|_{H}=\left.\pi \circ h\right|_{H}+\left.\pi \circ b\right|_{H} .
$$

Obviously $\left.\pi \circ h\right|_{H} \in Z^{1}(H, U)$ and $\left.\pi \circ b\right|_{H} \in \ell^{\infty}(H, U)$ since $\pi$ is continuous. By Corollary 1.5, $\left(q-\left.\varkappa(q)\right|_{H}\right) \in \ell^{\infty}(H, U)$, thus $q \in \ell^{\infty}(H, U)+Z^{1}(H, U)$.

The next lemma is a simplification of Dahmani et al [10, Theorem 2.23].

Lemma 5.2 Let $G \in \mathcal{X}$. Then for every $n \in \mathbb{N}$, there exists a subgroup $H_{n} \hookrightarrow_{h} G$ such that $H_{n} \cong F_{n} \times K$, where $F_{n}$ is the free group of rank $n$ and $K$ is finite.

We are now ready to prove Corollary 1.7.

Proof of Corollary 1.7 It is easy to see that the assumptions of Proposition 5.1 hold in the case $V=\ell^{p}(G)$ and $U=\ell^{p}(H)$. It is well known that $\operatorname{dim} E H_{b}^{2}(H)=\infty$ for every virtually free group which is not virtually cyclic (see. e.g., Hamenstädt [14]). To complete the proof it remains to note that every group $G \in \mathcal{X}$ contains a virtually free but not virtually cyclic hyperbolically embedded subgroup by Lemma 5.2. 
Stable commutator length Let $G$ be a group, and let $g \in[G, G]$. The commutator length of $g$, denoted $\mathrm{cl}_{G}(g)$, is defined as the minimal number of commutators whose product is equal to $g$ in $G$. The stable commutator length is defined by

$$
\operatorname{scl}_{G}(g)=\lim _{n \rightarrow \infty} \frac{\operatorname{cl}_{G}\left(g^{n}\right)}{n} .
$$

It is customary to extend $\operatorname{scl}_{G}$ to all elements $g$ for which have some positive power $g^{n} \in[G, G]$ by letting $\operatorname{scl}_{G}(g)=\operatorname{scl}\left(g^{n}\right) / n$. Basic facts and theorems about stable commutator length can be found in Calegari [7].

Following Calegari [7], we will denote space of quasimorphisms on $G$ by $\widehat{Q}(G)$. Recall that this is the same as $Q Z^{1}(G, \mathbb{R})$, where $\mathbb{R}$ is considered as a $G$-module with the trivial action. Note that in this setting Theorem 1.4 says that any antisymmetric quasimorphism on $H$ can be extended to a quasimorphism on $G$.

A quasimorphism $\varphi$ on $G$ is called homogeneous if for all $g \in G$ and all $n \in \mathbb{Z}$, $\varphi\left(g^{n}\right)=n \varphi(g)$. In particular, all homogeneous quasimorphisms are antisymmetric. We denote the subspace of homogeneous quasimorphisms by $Q(G)$. The connection between quasimorphisms and stable commutator length is provided by the Bavard Duality Theorem [1].

Theorem 5.3 (Bavard Duality Theorem) For any $g \in[G, G]$, there is an equality

$$
\operatorname{scl}_{G}(g)=\sup _{\varphi \in Q(G)} \frac{\varphi(g)}{2 D(\varphi)}
$$

where the supremum is taken over all homogeneous quasimorphisms of nonzero defect.

In fact, it is not hard to see that this supremum is always realized by some quasimorphism.

Given any quasimorphism $\varphi$, there is a standard way to obtain a homogeneous quasimorphism $\psi$, called the homogenization of $\varphi$. This is done by defining

$$
\psi(g)=\lim _{n \rightarrow \infty} \frac{\varphi\left(g^{n}\right)}{n} .
$$

Lemma 5.4 (Calegari [7, Corollary 2.59]) Let $\varphi \in \widehat{Q}(G)$ with homogenization $\psi$. Then $D(\psi) \leq 2 D(\varphi)$.

Our plan for proving Corollary 1.8 will be to take an element $h \in H$ and apply Bavard Duality to find a homogeneous quasimorphism which realizes (22) with respect to $\mathrm{scl}_{H}$. Then we can use Theorem 1.4 to extend this to a quasimorphism on all of $G$, 
then apply Bavard Duality again to find a lower bound on $\operatorname{scl}_{G}(h)$. In order to do this we will need to understand the defect of the extended quasimorphism.

Let $H$ be a group, and let $\xi: H \rightarrow H /[H, H] \otimes \mathbb{Q}$ be the natural map. A subset $Y \subseteq H$ will be called nice if $Y$ can be decomposed as $Y=Y_{1} \cup Y_{2}$ such that $\xi\left(Y_{1}\right)$ is linearly independent and $\left.\xi\right|_{Y_{2}} \equiv 0$.

Lemma 5.5 Every finitely generated subgroup of $H$ has a nice finite generating set.

Proof Let $H^{\prime}$ be a finitely generated subgroup of $H$, and let $X$ be a finite generating set of $H^{\prime}$. Then $\xi\left(H^{\prime}\right)$ is a finitely generated subgroup of a torsion-free Abelian group, and hence $\xi\left(H^{\prime}\right)$ is a finitely generated free Abelian group. Let $\left\{v_{1}, \ldots, v_{n}\right\}$ be a basis for $\xi\left(H^{\prime}\right)$ as a free Abelian group and let $y_{i} \in H^{\prime}$ be such that $\xi\left(y_{i}\right)=v_{i}$. Then for each $x \in X$, there exist integers $a_{x, 1}, \ldots, a_{x, n}$ such that $\xi(x)=\sum_{i=1}^{n} a_{x, i} v_{i}$. Let $\hat{x}=x y_{1}^{-a_{x, 1}}, \ldots, y_{n}^{-a_{x, n}}$. Now let $Y_{1}=\left\{y_{1}, \ldots, y_{n}\right\}$, and let $Y_{2}=\{\hat{x} \mid x \in X\}$. Then clearly $Y=Y_{1} \cup Y_{2}$ is nice, and $\langle Y\rangle=\langle X\rangle=H^{\prime}$.

Lemma 5.6 (Fuchs [12, Theorem 16.1]) Let $B$ be a subgroup of an Abelian group $A$, and let $D$ be a divisible Abelian group. Then every homomorphism from $B \rightarrow D$ can be extended to a homomorphism from $A \rightarrow D$.

The reason we are interested in nice subsets is the following lemma.

Lemma 5.7 For any group $H$, any nice finite subset $Y \subseteq H$, and any $\varphi \in Q(H)$, there exists $\varphi^{\prime} \in Q(H)$ such that $\left.\left.\varphi^{\prime}\right|_{[H, H]} \equiv \varphi\right|_{[H, H]}, D\left(\varphi^{\prime}\right)=D(\varphi)$, and for all $y \in Y$,

$$
\left|\varphi^{\prime}(y)\right| \leq 2 D\left(\varphi^{\prime}\right) \operatorname{scl}_{H}(y) .
$$

Proof Let $Y=Y_{1} \cup Y_{2}$ be the decomposition given by the definition of a nice subset. If $y \in Y_{2}$, then there exists some $n$ such that $y^{n} \in[H, H]$. Then for any $\varphi \in Q(H)$, Bavard Duality gives

$$
|\varphi(y)| \leq 2 D(\varphi) \operatorname{scl}_{H}(y) .
$$

Now, let $A=H /[H, H]$, and let $B$ be the image of $\langle Y\rangle$ inside $A$. Let $\theta$ be the quotient map $\theta: H \rightarrow A$. Then by definition of nice subsets we can define a homomorphism $\alpha: B \rightarrow \mathbb{R}$ such that $\alpha(\theta(y))=\varphi(y)$ for all $y \in Y_{1}$. Since $\mathbb{R}$ is divisible, Lemma 5.6 allows us to extend $\alpha$ to all of $A$. Composing $\alpha$ with $\theta$ gives a homomorphism $\beta: H \rightarrow \mathbb{R}$ which satisfies $\beta(y)=\varphi(y)$ for all $y \in Y_{1}$. Now we set $\varphi^{\prime}=\varphi-\beta$. Since $\beta$ vanishes on $[H, H],\left.\left.\varphi^{\prime}\right|_{[H, H]} \equiv \varphi\right|_{[H, H]}$. Since $\varphi^{\prime}$ is a shift of $\varphi$ by a 
homomorphism, $D\left(\varphi^{\prime}\right)=D(\varphi)$. Furthermore, combining the fact that $\varphi^{\prime}(y)=0$ for all $y \in Y_{1}$ with (23), we get that for all $y \in Y$,

$$
|\varphi(y)| \leq 2 D\left(\varphi^{\prime}\right) \operatorname{scl}_{H}(y) .
$$

We are now ready to prove Corollary 1.8 .

Proof of Corollary 1.8 Let $H \hookrightarrow_{h}(G, X)$, and by Lemma 2.3 there exists $Y^{\prime}$ a finite subset of $H$ such that the relative metric $\widehat{d}$ on $H$ is bi-Lipschitz equivalent to the word metric with respect to $Y^{\prime}$. By Lemma 5.5 the subgroup $\left\langle Y^{\prime}\right\rangle$ has a nice finite generating set $Y$. Let $d_{Y}$ be the word metric with respect to $Y$. Then $d_{Y}$ is bi-Lipschitz equivalent to the relative metric $\widehat{d}$ on $H$, so there exists a constant $L$ such that for all $f, g \in H$,

$$
d_{Y}(f, g) \leq L \widehat{d}(f, g) .
$$

Fix some $h \in[H, H]$, and let $\varphi \in Q(H)$ be the quasimorphism which realizes the Bavard Duality; that is, $\operatorname{scl}_{H}(h)=\varphi(h) /(2 D(\varphi))$. Let $\varphi^{\prime}$ be the modified quasimorphism provided by Lemma 5.7 .

Let $\iota: Q(H) \rightarrow \widehat{Q}(G)$ be map provided by Theorem 4.2 . Then by part $(b)$ of Theorem 4.2 we have

$$
D\left(\iota\left(\varphi^{\prime}\right)\right) \leq 54 K+66 D\left(\varphi^{\prime}\right),
$$

where $K$ is defined by $K=\max \left\{\left|\varphi^{\prime}(k)\right|: \widehat{d}(1, k)<15 C\right\}$. However, by (24) we get $K \leq \max \left\{\left|\varphi^{\prime}(k)\right|: d_{Y}(1, k)<15 C L\right\}$. Inductively applying the definition of a quasimorphism along with Lemma 5.7, for any such $k$ we get

$$
\left|\varphi^{\prime}(k)\right| \leq 15 C L\left(D\left(\varphi^{\prime}\right)+2 D\left(\varphi^{\prime}\right) \max _{y \in Y}\left\{\operatorname{scl}_{H}(y)\right\}\right) .
$$

That is, we have bound $K$ as a constant multiple of $D\left(\varphi^{\prime}\right)$. Thus there exists a constant $M$ (which is independent of $\varphi^{\prime}$ ) such that

$$
D\left(\iota\left(\varphi^{\prime}\right)\right) \leq 54 K+66 D\left(\varphi^{\prime}\right) \leq M D\left(\varphi^{\prime}\right) .
$$

Now, $\iota\left(\varphi^{\prime}\right)$ is a quasimorphism on $G$, and in order to apply Bavard Duality we homogenize $\iota\left(\varphi^{\prime}\right)$ to get a quasimorphism $\psi$, satisfying $D(\psi) \leq 2 D\left(\iota\left(\varphi^{\prime}\right)\right)$. Then applying the definition of $\psi$, along with the homogeneity of $\varphi^{\prime}$ and the conditions of Theorem 1.4 gives

$$
\psi(h)=\lim _{n \rightarrow \infty} \frac{\iota\left(\varphi^{\prime}\right)\left(h^{n}\right)}{n}=\varphi^{\prime}(h)=\varphi(h) .
$$


Also, (25) and Lemma 5.4 show that $D(\psi) \leq 2 D\left(\iota\left(\varphi^{\prime}\right)\right) \leq 2 M D\left(\varphi^{\prime}\right)=2 M D(\varphi)$. Applying Bavard Duality again gives

$$
\operatorname{scl}_{G}(h) \geq \frac{\psi(h)}{2 D(\psi)} \geq \frac{\varphi(h)}{4 M D(\varphi)}=\frac{1}{2 M} \operatorname{scl}_{H}(h) .
$$

Proof of Corollary 1.9 If $H$ is an almost malnormal quasiconvex subgroup of a hyperbolic group, then $G$ is hyperbolic relative to $H$ (Bowditch [5]). Hence $H$ is hyperbolically embedded in $G$ by Dahmani et al [10, Proposition 2.4] and the claim follows from Corollary 1.8.

Remark 5.8 Note that the malnormality condition can not be dropped in Corollary 1.9 even for free groups. For example, let $F=F(x, y, t)$ be the free group of rank 3 with basis $\{x, y, t\}$. In what follows we write $a^{b}$ for $b^{-1} a b$ and $[a, b]$ for $a^{-1} b^{-1} a b$. Let $H=\left\langle x, y, x^{t}, y^{t}\right\rangle$ and let

$$
h_{k}=[x, y]^{-k}\left[x^{t}, y^{t}\right]^{k} .
$$

Since the subset $\left\{x, y, x^{t}, y^{t}\right\} \subseteq G$ is Nielsen reduced, the subgroup $H$ is freely generated by $x, y, x^{t}, y^{t}$. Therefore $\operatorname{scl}_{H}\left(h_{k}\right)=k+1 / 2$ (see Calegari [7, Example 2.100]). On the other hand, we have

$$
\operatorname{scl}_{G}\left(h_{k}\right)=\operatorname{scl}_{G}\left([x, y]^{-k}\left([x, y]^{k}\right)^{t}\right)=\operatorname{scl}_{G}\left(\left[[x, y]^{k}, t\right]\right) \leq 1 .
$$

Thus $\operatorname{scl}_{H}\left(h_{k}\right) / \operatorname{scl}_{G}\left(h_{k}\right) \rightarrow \infty$ as $k \rightarrow \infty$.

\section{Acknowledgments}

We are grateful to Francois Dahmani and Ionut Chifan, with whom we discussed this project at various stages. This research was supported by the NSF grant DMS-1006345 and by the RFBR grant 11-01-00945.

\section{References}

[1] C Bavard, Longueur stable des commutateurs, Enseign. Math. 37 (1991) 109-150 MR1115747

[2] M Bestvina, K Bromberg, K Fujiwara, Bounded cohomology via quasi-trees arXiv: 1306.1542

[3] M Bestvina, M Feighn, A hyperbolic Out $\left(F_{n}\right)$-complex, Groups Geom. Dyn. 4 (2010) 31-58 MR2566300

[4] M Bestvina, K Fujiwara, Bounded cohomology of subgroups of mapping class groups, Geom. Topol. 6 (2002) 69-89 MR1914565 
[5] B H Bowditch, Relatively hyperbolic groups, Internat. J. Algebra Comput. 22 (2012) 1250016, 66 MR2922380

[6] R Brooks, Some remarks on bounded cohomology, from: "Riemann surfaces and related topics”, (I Kra, B Maskit, editors), Ann. of Math. Stud. 97, Princeton Univ. Press (1981) 53-63 MR624804

[7] D Calegari, scl, MSJ Memoirs 20, Mathematical Society of Japan, Tokyo (2009) MR2527432

[8] D Calegari, K Fujiwara, Stable commutator length in word-hyperbolic groups, Groups Geom. Dyn. 4 (2010) 59-90 MR2566301

[9] I Chifan, T Sinclair, On the structural theory of $\mathrm{II}_{1}$ factors of negatively curved groups, Ann. Sci. Éc. Norm. Supér. (4) 46 (2013) 1-33

[10] F Dahmani, V Guirardel, D Osin, Hyperbolically embedded subgroups and rotating families in groups acting on hyperbolic spaces arXiv:1111.7048

[11] D B A Epstein, K Fujiwara, The second bounded cohomology of word-hyperbolic groups, Topology 36 (1997) 1275-1289 MR1452851

[12] L Fuchs, Abelian groups, Publishing House of the Hungarian Academy of Sciences, Budapest (1958) MR0106942

[13] U Hamenstädt, Lines of minima in outer space arXiv:0911.3620

[14] U Hamenstädt, Bounded cohomology and isometry groups of hyperbolic spaces, J. Eur. Math. Soc. (JEMS) 10 (2008) 315-349 MR2390326

[15] U Hamenstädt, Isometry groups of proper CAT(0)-spaces of rank one, Groups Geom. Dyn. 6 (2012) 579-618 MR2961285

[16] R C Lyndon, P E Schupp, Combinatorial group theory, Ergeb. Math. Grenzgeb. 89, Springer, Berlin (1977) MR0577064

[17] I Mineyev, N Monod, Y Shalom, Ideal bicombings for hyperbolic groups and applications, Topology 43 (2004) 1319-1344 MR2081428

[18] N Monod, Continuous bounded cohomology of locally compact groups, Lecture Notes in Mathematics 1758, Springer, Berlin (2001) MR1840942

[19] N Monod, An invitation to bounded cohomology, from: "Proc. ICM, Vol. II", (M Sanz-Solé, J Soria, J L Varona, J Verdera, editors), Eur. Math. Soc., Zürich (2006) 1183-1211 MR2275641

[20] N Monod, Y Shalom, Negative curvature from a cohomological viewpoint and cocycle superrigidity, C. R. Math. Acad. Sci. Paris 337 (2003) 635-638 MR2030102

[21] N Monod, Y Shalom, Orbit equivalence rigidity and bounded cohomology, Ann. of Math. 164 (2006) 825-878 MR2259246

[22] D V Osin, Elementary subgroups of relatively hyperbolic groups and bounded generation, Internat. J. Algebra Comput. 16 (2006) 99-118 MR2217644 
[23] D Osin, Small cancellations over relatively hyperbolic groups and embedding theorems, Ann. of Math. 172 (2010) 1-39 MR2680416

[24] S Popa, Deformation and rigidity for group actions and von Neumann algebras, from: "Proc ICM, Vol. I", (M Sanz-Solé, J Soria, J L Varona, J Verdera, editors), Eur. Math. Soc., Zürich (2007) 445-477 MR2334200

[25] A Sisto, Contracting elements and random walks arXiv:1112.2666

[26] A Thom, Low degree bounded cohomology and $L^{2}$-invariants for negatively curved groups, Groups Geom. Dyn. 3 (2009) 343-358 MR2486803

Department of Mathematics, Vanderbilt University 1326 Stevenson Center, Nashville, TN 37240, USA

michael.b.hull@vanderbilt.edu, denis.osin@gmail.com

http://math.vanderbilt.edu/people/hull,

http://math.vanderbilt.edu/people/osin

Received: 26 May 2012 Revised: 28 January 2013 